\title{
LA MISSIONARIETÀ DELLA CHIESA NELL'ENCICLICA REDEMPTORIS MISSIO DI GIOVANNI PAOLO II DI FRONTE ALLE VOCI DEL SINODO AMAZZONICO
}

\begin{abstract}
Oggetto di questo studio è la questione della natura missionaria della Chiesa, prospettata e rinnovata con più profonda e convincente impostazione come espresso nell'enciclica Redemptoris missio di san Giovanni Paolo II. Il XXX ${ }^{\circ}$ anniversario della sua promulgazione (7 dicembre 1990) costituisce una ragione particolare per riprendere l'argomento e trattarlo nell'attuale contesto storico-ecclesiale. L'esposizione e l'approfondita ricerca dimostrano l'attualità dei contenuti presenti nel documento e la permanente validità del mandato di Gesù affidato agli apostoli e a tutta la Chiesa di andare nel mondo intero per annunciare il Vangelo e portare il battesimo della salvezza a tutti i popoli. La necessità di riportare all'attenzione questo tema è determinato anche dalla condizione interna della Chiesa stessa che sembra pian piano abbandonare o non considerare con la dovuta serietà il mandato di cui fu investita sin dalle sue origini. Di conseguenza, di fronte ad un indebolimento della consapevolezza missionaria all'interno della Chiesa, riportare e rievocare senza equivoci la questione del compito di evangelizzare appare un impegno necessario.
\end{abstract}

\section{INTRODUZIONE}

La Redemptoris missio si iscrive nell'insieme del magistero ecclesiale conciliare e post-conciliare, soprattutto a partire dal Decreto sull'attività missionaria della Chiesa Ad gentes Concilio Vaticano $\mathrm{II}^{1}$, che chiarisce e ribadisce, insieme

Prof. padre Maksym Adam Kopiec OFM - Pontificia Università Antonianum a Roma (20042019); e-mail: maksymk@libero.it; ORCID: 0000-0002-1055-6251.

1 Concilio Vaticano II, Decreto sull'attività missionaria della chiesa "Ad gentes", in: Enchiridion Vaticanum (=EV), Bologna $2002^{18}, 1 / 1087-1242$. 
all'insegnamento dei papi Paolo $\mathrm{VI}^{2}$, Benedetto $\mathrm{XVI}^{3}$ e Francesco ${ }^{4}$, la natura missionaria della Chiesa di Cristo. L'enciclica di papa Wojtyła evidenzia la permanente validità del mandato missionario - attestato chiaramente nel Nuovo Testamento $^{5}$ - cioè, che Cristo dopo la sua Risurrezione, in occasione delle apparizioni e infine della Sua gloriosa ascensione al cielo e il ritorno al Padre, affidò agli apostoli, ai loro successori e a tutta la comunità della Chiesa il mandato di andare in tutto il mondo e di annunciare il Vangelo a tutti popoli, battezzandoli nel nome del Padre, e del figlio e dello Spirito Santo - come riferiscono tutti i sinottici ${ }^{6}$, l'evangelista Giovanni ${ }^{7}$ e l'autore degli Atti degli Apostoli ${ }^{8}$.

La validità di questo mandato emerge con maggior urgenza in questi giorni in occasione dell'appena concluso Sinodo Amazzonico svoltosi a Roma, dal 6 al 27 ottobre del 2019. Anche se il Sinodo non ha nessuna facoltà decisiva e non entra nel magistero ufficiale della Chiesa nelle questioni del magistero della Chiesa, comunque viene considerato come 'corpo ausiliare' con i suoi suggerimenti, proposte, osservazioni ecc. La peculiarità di questo Sinodo stava nel fatto di voler affrontare certe questioni di natura teologico-disciplinare riguardanti la Chiesa universale ${ }^{9}$, determinando geo-politicamente ed ideologicamente la prospettiva di trattarle. Il grosso rischio dei temi discussi nell'aula sinodale era, in fin dei conti, quello della necessità o, nel migliore dei casi, del modo di evangelizzare i popoli e le culture che vivono ancora nell'ignoranza del Vangelo di Cristo o della sua mistificata conoscenza. In questo contesto il bisogno di richiamarsi alla Redemptoris missio, data la ricorrenza di 30 anni dalla sua promulgazione (7 dicembre 1990), sembra una cosa del tutto dovuta.

\section{LA CHIESA, MISSIONARIA PER SUA NATURA, NELLA REDEMPTORIS MISSIO}

L'esposizione sarà divisa in due momenti (in pratica, in due articoli). In questa istanza, la considerazione teologica, sviluppata in maniera diacronica, punta piut-

\footnotetext{
Paolo VI, Esortazione apostolica postsinodale "Evangelii nuntiandi” (1975), EV 5/1588-1716. Benedetto XVI, L'esortazione apostolica “Verbum Domini” (2010), EV 26/2218-2433.

Francesco, L'esortazione apostolica “Evangelii gaudium”, AAS 12 (2013) 1019-1137.

Seminario Urbano, La missione nella Bibbia, 16 aprile 1996, http://www.cistercensi.info/monari/1996/m19960416.htm.

6 Mt 28,18-20; Mc 16,15-20; Lc 24,46-53.

7 Gv 17,17-19; 20,21-22.

9 Innanzitutto si trattava di tre argomenti: il sacerdozio dei diaconi sposati, il diaconato delle donne e il rito liturgico amazzonico.
}

8 At 1,8 . 
tosto sull'opera evangelizzatrice come quella che per natura spetta alla Chiesa. Essa sarà messa in rilievo riportando il modello della vita missionaria, lasciato dall'autore dell'enciclica, cioè da papa Giovanni Paolo II, uno dei più grandi apostoli del cristianesimo degli ultimi secoli. Successivamente si rifletterà del giusto rapporto tra la dimensione verticale e orizzontale della missione della Chiesa tenendo conto, in seguito, di alcune proposte del Sinodo, viste come sfide per riconfermare l'autentica natura missionaria della Chiesa, istituita come tale da Dio stesso.

\section{LA CONTINUITÀ TEOLOGICA E UN'ADEGUATA ERMENEUTICA DEL TESTO}

La Redemptoris missio ${ }^{10}$ a differenza della Evangelii nuntiandi e della recente Evangelii gaudium che sono Esortazioni è una Enciclica e quindi ha un maggior spessore magisteriale. È stata scritta nel XXV anniversario del Decreto Ad gentes del Concilio Vaticano II. Cinque anni fa, invece, nell'anno 2015 abbiamo ricordato il $\mathrm{XXV}^{\circ}$ della pubblicazione della stessa Redemptoris missio. Il documento di Giovanni Paolo II, contro una qualsiasi scettica opinione, fino ad oggi non ha perso nulla della sua attualità, anzi Evangelii gaudium rappresenta la ripresa e il tentativo di contestualizzare i suoi contenuti più essenziali. Questa enciclica non è soltanto un gesto celebrativo del $\mathrm{XXV}^{\circ}$ del Decreto missionario del Concilio Vaticano II, come sta scritto nell'Introduzione e nella data finale, ma è un vero grido del papa, che invita tutta la Chiesa a riconsiderare lo specifico impegno missionario e ad impegnarsi per l'evangelizzazione del mondo non cristiano. Giovanni Paolo II incoraggia gli uomini ad aprirsi a Cristo e chiede alla Chiesa il coraggio di testimoniarlo e di proporlo al mondo d'oggi. Questo infatti è il primo dovere assegnato da Cristo alla sua comunità, è il primo servizio offerto per la crescita integrale dell'umanità, è il modo più sicuro per il rinnovamento della vita cristiana superando le tensioni interne, è un'esigenza di condivisione dei doni divini ricevuti, ma è anche un diritto di scelta da parte dei singoli e dei popoli.

In questo momento così difficile, l'invito del papa perché la Chiesa intera si rinnovi nell'impegno per realizzare il mandato del Signore suona molto diverso da tante propagande. Le comunità cattoliche sono chiamate al loro dovere di testimoniare e annunciare il Cristo nella carità fattiva ed effettiva verso tutti, nel rispetto delle persone e delle culture, nella promozione della libertà da parte degli individui e dei popoli. Questi atteggiamenti, fondamenti della pace, sono conna-

10 Giovanni Paolo II, Lettera enciclica “Redemptoris missio” (=RMi), EV 12 (1990), 547-732. 
turali a tutto il messaggio cristiano che è la rivelazione dell'amore di Dio per noi. L'annuncio del Vangelo non può essere opprimente, perché è per sua natura liberatorio; non può essere imposto ma proposto. Ad imitazione di Cristo, la Chiesa missionaria non condanna nessuno a morte, ma si lascia mettere in croce, come espressione culmine dell'amore e della salvezza. Gli esempi di missionari testimoni di Cristo fino al martirio, sono notizie continue anche in tempi recenti ${ }^{11}$. "Al termine del secondo millennio, la Chiesa è diventata nuovamente Chiesa di martiri. Le persecuzioni nei riguardi dei credenti - sacerdoti, religiosi, laici - hanno operato una grande semina di martiri in varie parti del mondo" 12 .

Infatti, come dice Rino Fisichella, per paradossale che possa sembrare, i martiri dei nostri giorni - dei tempi di libertà religiosa, rispetto, tolleranza, dialogo, apertura ecc. - sono giovani, ragazzi e ragazze, uomini e donne di ogni età, ancora uccisi, violentati, torturati, scherniti ed emarginati solo perché cristiani. Quanti nomi potrebbero riempire un nuovo e aggiornato martirologio dei nostri tempi. Purtroppo, il secolo XX ha visto un numero di martiri cristiani superiore a quello dei diciannove secoli che ci hanno preceduto. L'ingresso nel XXI secolo, tristemente, sta allungando l'elenco senza lasciar intravedere una diminuzione o almeno qualche tregua. Ciò che rende spesso inconcepibile questa situazione è il silenzio di tante voci sul palcoscenico della vita pubblica - anche tra le autorità della Chiesa stessa d'oggi -, quel silenzio che essendo complice per l'incapacità di debellare la violenza, diventa ancor più udibile e percettibile ${ }^{13}$.

Nonostante tutto ciò, l'enciclica è radicata nella visione del Vaticano II, non solamente per le basi teologiche e per le aperture pastorali, ma per l'ottimismo ${ }^{14}$ che la domina e che è stato espresso quasi profeticamente dallo stesso papa: "Vedo albeggiare una nuova epoca missionaria, che diventerà giorno radioso e ricco di frutti, se tutti i cristiani e, in particolare, i missionari e le giovani chiese risponderanno con generosità e santità agli appelli e alle sfide del nostro tempo" 15 . Malgrado le diverse difficoltà e contro ogni tentazione di scoraggiamento e di pessimismo, il papa offre dunque una visione ottimistica e piena di speranza. Questo ottimismo viene dalla fede ${ }^{16}$. Egli parla di "nuova primavera del cristianesimo",

11 Cfr. P. Gheddo, L'Ad gentes in Giovanni XXIII e Giovanni Paolo II, (25.05.2014), http://www. gheddopiero.it/index.php/lad-gentes-in-giovanni-xxiii-e-giovanni-paolo-ii-radio-maria-2014/.

12 Giovanni Paolo II, Lettera apostolica “Tertio Millennio Adveniente”, EV 14 (1994) 1781, 37.

13 Cfr. R. Fisichella, I segni del giubileo. Il pellegrinaggio, la città di Pietro e Paolo, la Porta Santa, la professione di fede, la carità, l'indulgenza, Cinisello Balsamo 2015.

14 Cfr. M. Lemonnier, Svolte storiche della missione, in: AA.Vv., La missione del redentore. Commenti all'enciclica "Redemptoris missio", Bologna 1991, 27.

15 RMi 92.

16 Cfr. J. Tomko, La "Magna charta" per la missione del duemila, in: AA.Vv., La missione del redentore. Commenti all'enciclica "Redemptoris missio"..., 9. 
di "una umanità più preparata alla semina evangelica" 17 e ribadisce la convinzione che "Dio sta preparando una grande primavera cristiana, di cui già si vede l'inizio"18. Dopo venticinque anni - dalla chiusura del Concilio Vaticano II - di cammino travagliato del mondo e della Chiesa, il documento traccia il punto della situazione nei riguardi della missione. Ha ereditato dal Concilio una coraggiosa dinamica e una visione aperta, facendo però un discernimento delle posizioni teoriche e pratiche nella Chiesa. Ne risulta un "manifesto per la missione", una sintesi teoretico-operativa per la Chiesa del prossimo millennio ${ }^{19}$.

Nella Redemptoris missio si ritrovano le grandi linee dell'insegnamento e dell'attività pastorale di Giovanni Paolo II. Già il titolo richiama la sua prima enciclica programmatica (Redemptor hominis). Del resto le sue otto encicliche precedenti vi ritrovano un riscontro puntuale. Quelle antropologico-trinitarie (Redemptor hominis, Dives in misericordia, Dominum et vivificantem) costituiscono il punto di partenza dei tre primi capitoli, che sono di natura teologica e che rispondono alle grandi tematiche attuali che rafforzano la sollecitudine missionaria della Chiesa d'oggi, come l'unicità e l'universalità della salvezza in Gesù Cristo (cap. I), del Regno di Dio in rapporto a Cristo, alla Chiesa e quindi all'attività missionaria (cap. II), della presenza attiva dello Spirito nella Chiesa e nel mondo (cap. III). Anche se il respiro teologico è consistente, comunque vengono profondamente trattati gli aspetti pratici che sono importanti e innovativi. $\mathrm{Su}$ questo piano la Redemptoris missio proporrà di promuovere l'impegno missionario ad gentes, armonizzando le diverse attività invece di escluderle, dando priorità all'annuncio, verso il quale tutto tende. In tal modo i successivi capitoli (IV-VIII), più di carattere operativo, vanno letti nel loro rapporto con la dottrina sociale della Chiesa, esposta e sviluppata negli altri documenti del magistero di Giovanni Paolo II (Laborem exercens, Sollicitudo rei socialis). È anche da notare che la pubblicazione dell'enciclica precede immediatamente l'inizio dell'Anno della Dottrina Sociale della Chiesa proclamato il 1 gennaio $1991^{20}$ e che avrà la sua particolare espressione con la promulgazione di un'altra enciclica Centesimus annus, 1 maggio 1991. L'orizzonte operativo in cui si colloca la seconda parte dell'enciclica precisa dunque la verità degli impegni della Chiesa, origina

RMi 2-3.

18 RMi 86.

19 Cfr. M. Zago, Riscoprirci tutti missionari. La "Redemptoris missio": un orientamento per la lettura, www.indaco-torino.net/gens/34gens91.html [“Gen's - Rivista di vita ecclesiale" 34 (1991) 4].

20 Voglio quindi proclamare quello che oggi s'avvia "Anno della Dottrina Sociale della Chiesa", invitando con ciò i fedeli, nel contesto della commemorazione dell'Enciclica "Rerum novarum", a meglio conoscere, approfondire e diffondere l'insegnamento della Chiesa in materia sociale, "Insegnamenti" XIV (1995) 1, 1-5. 
ti dal suo essere "connaturalmente" missionaria: la cura pastorale di coloro che si riconoscono cristiani; la nuova evangelizzazione per coloro che non sono più o non si riconoscono cristiani; e infine l'attività missionaria verso i gruppi e i popoli che non sono mai stati cristiani. La missione ad gentes è poi chiarita da tre approcci convergenti: quello geografico, quello sociale, collegato ai nuovi fenomeni sociali, e quello culturale del rapporto con i moderni «areopaghi» (cap. IV). Il rapporto tra il dovere missionario di tutta la Chiesa e di ogni Chiesa locale e i "carismatici della missione" è un altro argomento trattato profondamente dall'enciclica ${ }^{21}$. Infatti l'attenzione alle giovani Chiese accompagna tutto il documento. L'avvenire della missione dipenderà in gran parte dal loro impegno missionario nel proprio ambiente e in tutto il mondo ${ }^{22}$. L'attività missionaria si realizza in un clima di rispetto e di dialogo. Il dialogo, talvolta, è la sola espressione possibile e sufficiente di una presenza missionaria. La promozione umana, l'impegno per la pace e la giustizia, l'inculturazione sono parti integranti della missione, ma per natura propria tendono all'annuncio della Buona Novella; questo annuncio costituisce il cuore, il centro dinamico e l'apice di tutte le attività missionarie (cap. V) ${ }^{23}$. Non c'è missione senza missionari, e d'altra parte non ci sono missionari senza comunità coinvolte nella cooperazione e nella testimonianza della propria incorporazione a Cristo (cap. VI e VII). La connessione tra santità e missione, non solo per i missionari - "i santi sono gli autentici missionari" -, ma anche per tutta la Chiesa, è un'altra sottolineatura importantissima. L'impegno missionario rinnova la Chiesa; e la santità dei cristiani è l'anima del suo dinamismo missionario (cap. VIII) ${ }^{24}$.

\section{REDEMPTORIS MISSIO E L'ATTIVITÀ MISSIONARIA DI GIOVANNI PAOLO II}

Il Papa ha rilanciato la "missione alle genti" in modo teologico e programmatico nell'enciclica Redemptoris missio, ma in modo concreto e pratico l'ha resa

${ }_{21}$ Cfr. D. Colombo, Mandati ad gentes ieri, oggi e sempre, in: AA.Vv., La missione del redentore. Commenti all'enciclica "Redemptoris missio"..., 75-87.

22 Cfr. M. Zago, Riscoprirci tutti missionari. La "Redemptoris Missio": un orientamento per la lettura, www.indaco-torino.net/gens/34gens91.html ["Gen's - Rivista di vita ecclesiale" 34 (1991) 4].

23 Cfr. M. Fitzgerald, Religioni in dialogo nel segno di Assisi, in: AA.Vv., La missione del redentore. Commenti all'enciclica "Redemptoris missio"..., 102-107; cfr. M. Fitzgerald, Dialogo interreligioso. Il punto di vista cattolico, Cinisello Balsamo 2007, 29-52.

24 Cfr. M. Zago, L'urgenza di tutte le vocazioni missionarie, in: AA.Vv., La missione del redentore. Commenti all'enciclica "Redemptoris missio"..., 88-91. 
realizzabile nei suoi 62 viaggi missionari extra-europei, di cui circa 40 in Europa (fino alla promulgazione dell'enciclica), quando ha rinfrancato e incoraggiato le giovani Chiese in paesi non cristiani. Si diceva che il Papa viaggiava troppo, ma chi diceva questo non è stato nei paesi da lui visitati dopo un suo viaggio. Inoltre richiamandosi a Paolo VI ha detto a Loreto nel 1994 durante l'incontro personale con i vescovi italiani:

I viaggi, le visite, sembra che oggi il papa deve essere presente non solamente spiritualmente, ma anche personalmente in diverse parti del mondo, e così io l'ho capito da Paolo VI. Se lui con la sua salute, che era piuttosto debole, poteva fare tutto questo, questo papa giovane più atletico, $[\ldots]$ deve anche lui avere il coraggio di andare in tutto il mondo, così ci ha detto Cristo, non ci ha detto 'sedete in Vaticano', ma ci ha detto: 'andate in tutto il mondo!' cioè, ai confini della terra ${ }^{25}$.

Il titoletto "Giovanni Paolo II è il centravanti delle missioni" introduce quanto ha detto padre Schiavone, un missionario domenicano toscano, che nel 1982 era in Pakistan da una quarantina d'anni. Secondo la sua testimonianza il Papa l'anno precedente aveva fatto una visita a Karachi, allora capitale del Pakistan, e aveva suscitato un grande entusiasmo nello stadio cittadino pieno di giovani musulmani ad applaudirlo. Diceva Schiavone: "Noi missionari che siamo in questo paese da decine d'anni, tollerati e a volte perseguitati, non avevamo mai nemmeno immaginato di poter essere testimoni di una scena simile: una folla di musulmani che applaudiva il nostro Papa! Abbiamo pianto di gioia". E concludeva dicendo: "Noi missionari abbiamo trovato il nostro centravanti!". Un caso simile racconta il cardinale Robert Sarah sull'accoglienza di Giovanni Paolo II in Guinea (sotto il governo dittatoriale di Sékou Touré) sia dai cristiani che dai musulmani; questi ultimi spiegano così la loro presenza e il loro saluto nei confronti del papa al suo arrivo: "Al tempo della rivoluzione siamo stati forzati a venire ad accogliere i dirigenti dell'URSS; non c'è alcun motivo per cui non dobbiamo uscire nelle strade per un grande credente e un uomo di Dio!"26.

Secondo A. Riccardi il papa slavo è una grande figura del Novecento, di cui esprime appieno la storia. È anche un personaggio del Duemila: si è spento nel nuovo secolo già iniziato e la sua ricchissima eredità religiosa, teologica e pratica deve continuare ad essere un punto di riferimento fermo. Testimone del complesso crocevia polacco e protagonista della scena mondiale per ventisette anni, Karol Wojtyła è stato un personaggio decisivo della vicenda religiosa contemporanea,

25 A. Ambrogetti, Il mistero dei 12. I vescovi del mondo a tavola con Giovanni Paolo II, Tau Editrice 2014, 91.

26 Cfr. R. Sarah, Dio o niente. Conversazione sulla fede con Nicolas Diat, Siena 2015, 89-90. 
ma anche un leader che ha collocato la Chiesa nel cuore e sul palcoscenico della storia mondiale e della vita sociale, un leader globale che ha toccato le fibre di tanti popoli e nazioni ${ }^{27}$.

Giovanni Paolo II ha lottato con tutte le sue forze, affinché l'Europa ritrovasse l'unità riconoscendo le sue radici cristiane ${ }^{28}$ - come quando lanciava l'appello di inserire il riferimento alla cristianità nella Preambola nella Costituzione Europea ${ }^{29}$ - ma capiva che la civiltà fondata ed edificata sul fondamento dei valori cristiani, a partire dai tempi moderni non aveva più la forza e la gioia della fede per portare Cristo ai miliardi di uomini e donne che ancora non lo conoscono ${ }^{30}$. Aveva una visione profetica della missione e viaggiava il più possibile nelle giovani Chiese, proprio per promuovere il primo annunzio e il dialogo interreligioso chiamando $\mathrm{i}$ giovani e le giovani Chiese ad esserne protagonisti. Alla $\mathrm{X}^{\mathrm{a}}$ Giornata Mondiale della Gioventù a Manila (5-10 gennaio 1995) ha gridato: "A ciascuno di voi Cristo dice: 'Io mando voi'. Ecco l'ideale del giovane cristiano: sentirsi mandato da Cristo, avere nella vita lo scopo ben preciso di realizzare il mandato di Cristo. Non si può vivere senza ideali. I giovani (ma anche tutti quanti) hanno bisogno di dare senso ed entusiasmo alla vita, proporsi grandi mete, sentirsi protagonisti di grandi conquiste, spaziare per grandi orizzonti. Ecco, la missione è tutto questo". "Il mondo è stanco delle vecchie ideologie" ha detto visitando Cuba nel 1998 e riferendosi, tra l'altro, al comunismo, secolarismo, neoliberalismo economico, consumismo, vuoto spirituale, nichilismo e spiegava che la missione non è un'ideologia, ma un ideale che risponde al vuoto e alla più profonda ricerca umana e che viene dall'avvenimento che ha cambiato il corso della storia: la persona di Gesù Cristo unico Salvatore dell'uomo.

La dimensione missionaria è essenziale per essere cristiani autentici invitando gli altri alla fede. Aggiungeva pure che la Chiesa non può non essere missionaria. Ma come? La missionarietà va intesa in due modi principali. Anzitutto verso i non cristiani, soprattutto le masse umane dell'Asia, che ancora attendono di conoscere la Buona Notizia della nascita del Salvatore. La Chiesa ha bisogno di missionari per annunziare Cristo alle genti, quelle che, come ha detto il papa, "anelano all'autentica liberazione e realizzazione. I poveri cercano giustizia e solidarietà; gli oppressi chiedono libertà e dignità; i ciechi invocano la luce e la verità". La missione

27 A. Riccardi, Giovanni Paolo II Santo. La biografia, Cinisello Balsamo 2014, 590.

28 Giovanni Paolo II, Esortazione apostolica post-sinodale "Ecclesia in Europa", 1-22, 28 giugno 2003, http://w2.vatican.va/content/john-paul-ii/it/apost_exhortations/documents/hf_jp-ii_ exh_20030628_ecclesia-in-europa.html.

29 Cfr. C.M. Martini, L'Europa interpellata dalla Redemptoris missio, in: AA.Vv., Riflessioni sulla "Redemptoris missio", Roma 1991, 237-247.

30 Cfr. M. Invernizzi, L'Europa nel magistero della Chiesa, "Christianità" 396 (2019) marzo-aprile, 5-17. 
verso i non cristiani è la giovinezza della Chiesa. C'è anche un secondo modo di realizzare la missione: "Essere missionari nella nostra società". C'è una "missione nel quotidiano" a cui dobbiamo educarci: testimoniare e annunziare Cristo con la nostra vita nella famiglia, nella società, nella scuola, sul lavoro, in politica. Ma per esercitare questa missione dobbiamo andare controcorrente (il Vangelo è sempre all'opposizione rispetto allo spirito del mondo). Il papa, nel discorso della veglia di preghiera a Manila, ha ammonito i giovani con forza: "Attenti ai falsi maestri! Appartengono alle élites intellettuali della scienza, della cultura e dei mass media. Loro presentano un anti-vangelo che dichiara morto ogni ideale. Vogliono che voi siate come loro: dubbiosi e cinici". "Chi ci ha rubato le ali?" chiedeva un giovane filippino. Il Papa a Manila ha proposto, per volare, due ali ai giovani credenti: fede e missione ${ }^{31}$.

\section{LA MISSIONARIETÀ E L'IMPEGNO SOCIALE DI GIOVANNI PAOLO II}

La missione della Chiesa è quella di annunciare e portare in tutto il mondo il Vangelo della libertà e della liberazione da ogni forma di schiavitù, di alienazione, di qualsiasi ostacolo che impedisce all'uomo di realizzarsi pienamente e in tutta la sua dignità come persona umana creata a immagine e somiglianza di Dio e redenta dal Figlio di Dio fattosi uno di noi. In questa ottica si comprende quell'ardore di Giovanni Paolo II che lo accompagnava nei viaggi per dare a tutti un messaggio di liberazione. Un eclatante esempio è quello di Puebla in Messico nel gennaio 1979, quando ha aperto l'Assemblea del CELAM (Consiglio Episcopale dell'America latina e dei Caraibi). Il documento di preparazione era impostato sul tema "Vedere, Giudicare, Agire", che portava ai problemi economico-politico-sociali: "vedere" la situazione dei popoli dell'America Latina, "giudicare" di chi è la colpa e poi "agire" per liberare i popoli da ogni oppressione. Il Papa, nel discorso iniziale dice che lo schema di preparazione va cambiato: "Per liberare i popoli latino-americani, ripartiamo da Cristo". Giovanni Paolo II era profondamente innamorato di Gesù Cristo, di cui parlava come una persona viva che egli aveva incontrato e di cui si era innamorato. Ai vescovi e sacerdoti dell'America Latina diceva: "Lasciatevi prendere dall'amore di Cristo; lasciatevi penetrare, coinvolgere, illuminare e cambiare dall'amore di Cristo; nella misura in cui sarete tutti di Cristo, sarete anche tutti degli uomini”. Riaffermava chiaramente che la missione della Chiesa è di natura religiosa, portare la salvezza in Cristo, liberando l'uomo prima dal

31 Cfr. P. Gheddo, http://www.gheddopiero.it/index.php/lad-gentes-in-giovanni-xxiii-e-giovanni-paolo-ii-radio-maria-2014/. 
peccato personale e poi cambiando la società oppressiva attraverso l'azione e la testimonianza dei credenti in Cristo.

La Chiesa, portando il Vangelo di Gesù, si schiera inequivocabilmente dalla parte dei poveri. Pertanto riconoscendo il primato della conversione a Cristo nel senso verticale, insisteva anche sulla fraternità e solidarietà universale in senso orizzontale, proclamava di dover portare alla ribalta tutte le sofferenze e le ingiustizie del mondo, gli sfruttamenti, le oppressioni, le persecuzioni che cercano di rendere l'essere umano una "non-persona", "un insignificante", "un oggetto" ed annunciare, per contro, la liberazione integrale, evangelica della persona umana e della sua dignità ${ }^{32}$. Questa idea è intrinsecamente legata alla "nuova evangelizzazione" alla cui base c'è irrinunciabilmente la solidarietà verso i poveri; solidarietà che ha trovato la sua articolazione nella nota espressione "opzione preferenziale per i poveri" presente in tanti documenti di Giovanni Paolo II in cui trattava e sviluppava la dottrina sociale della Chiesa ${ }^{33}$. In essi emerge la visione dell'uomo liberato nella sua totalità e ri-dotato della sua dignità; questo il papa lo confermava in modo pratico e concreto visitando 13 volte i vari villaggi della popolazione indigena in America Latina, parlando ai "favelados" di Rio de Janeiro, ai lebbrosi di Marituba in Amazzonia, agli indios di Oaxaca in Messico o ai pescatori di Baguio nelle Filippine; quando condannava con forza ogni violazione dei diritti dell'uomo davanti a dittatori come F. Marcos (Filippine), A. Pinochet (Cile), A. Stroessner (Paraguay), Mobutu Sese Seko (Zaire), Fidel Castro (a Cuba), i Sandinisti (in Nicaragua) ${ }^{34}$. Per dimostrare l'amore di Cristo in modo concreto è stato convocato il Pontificio Consiglio Cor Unum al fine di aiutare i popoli di tutto il mondo che vivono nell'estrema miseria, povertà e sofferenza. È da notare che nella stragrande maggioranza dei casi i paesi aiutati sono quelli con popolazione non cristiana, spesso con maggioranza musulmana ${ }^{35}$.

In Messico Giovanni Paolo II ha preso solennemente le difese degli indios. A Oaxaca un indio gli dice: "Santità, noi viviamo peggio delle vacche e dei porci. Abbiamo perso le nostre terre, noi che eravamo liberi, ora siamo schiavi". Il papa si stringe la testa fra le mani e rispondendo dice: "Il papa sta con queste masse di indios e di contadini, abbandonate ad un indegno livello di vita, a volte sfruttate

32 Basta menzionare solo alcune encicliche: Redemptor hominis, Laborem exercens, Sollicitudo rei socialis, Centesimus annus, Veritatis splendor, Evangelium vitae: Tutte le encicliche di Giovanni Paolo II, Paoline Editoriale Libri, Milano 2010².

33 Per una sintesi della dottrina sociale da Leone XIII fino a Benedetto XVI, si consiglia M. Ormas, Umanesimo cristiano e modernità. Introduzione alle Encicliche sociali. Dalla "Rerum novarum" alla "Caritas in veritate", Città del Vaticano 2014.

34 Cfr. G. Mazzoleni, L'eredità missionaria di Giovanni Paolo II, www.consolata.org/.../13273-leredita-missionaria-di-giovanni-paolo-ii.

35 Cfr. R. Sarah, Dio o niente. Conversazione sulla fede con Nicolas Diat..., 179. 
duramente. Ancora una volta gridiamo forte: rispettate l'uomo! Egli è l'immagine di Dio! Evangelizzate perché questo diventi realtà, affinché il Signore trasformi i cuori ed umanizzi i sistemi politici ed economici, partendo dall'impegno responsabile dell'uomo". Inoltre non si dimentichi la sua richiesta ai paesi ricchi di cancellare i debiti esteri dei popoli poveri nell'anno 2000 in occasione del grande giubileo, inteso nel senso biblico del termine ${ }^{36}$. Nemmeno si deve scordare che è stato Giovanni Paolo II a far costruire sul territorio dello Stato Vaticano la casa per i poveri gestita dalle suore della Congregazione delle Missionarie della Carità fondata dalla beata Teresa di Calcutta. Gesti e fatti pieni di significato.

Sul piano politico e diplomatico voleva realizzare la vocazione missionaria attraverso e nella forma del dialogo. Basta pensare all'insistenza con cui cercava di instaurare e poi sviluppare i contatti di dialogo con i vari capi degli stati e delle nazioni, come ad esempio, con il capo dello Stato Sovietico M. Gorbačëv. Simili contatti di dialogo ha realizzato anche quando parlava del valore della cultura africana (in Benin) e dello "sviluppo dal volto umano" (in Gabon); egli incideva fortemente sulle coscienze dei popoli. Quante volte un popolo sofferente e umiliato (si pensi alla Guinea Equatoriale appena uscita dalla spaventosa dittatura di Macias Nguema) ha ricevuto dalla visita del papa il provvidenziale stimolo a riprendere con coraggio la via della riconciliazione e della ricostruzione ${ }^{37}$.

\section{LE SFIDE DELLA MISSIONE CRISTIANA E LA NECESSITÀ DELL'ENCICLICA}

Anche se il papa esprimeva ottimismo riguardo all'opera missionaria che stava vivendo la sua "nuova primavera", tuttavia affermava già dall'inizio che "la missione di Cristo redentore, affidata alla Chiesa, è ancora ben lontana dal suo compimento" e "che tale missione è ancora agli inizi" ${ }^{38}$. Inoltre sono ben evidenti i fattori negativi che causano perfino il rallentamento dell'evangelizzazione. Non mancano secondo il papa le difficoltà interne ed esterne che indeboliscono lo slancio missionario della Chiesa verso i non cristiani e questo deve preoccupare tutti i credenti in Cristo $^{39}$. Tra gli ostacoli esterni si potrebbero individuare: la ra-

\footnotetext{
36 Non sarebbe stato opportuno in occasione dell'anno Santo della Misericordia 2016 di rifare lo stesso appello ai paesi ricchi, ai loro capi e governi? Invece non si è sentito nulla circa una iniziativa del genere da parte della Santa Sede.

37 Cfr. P. Gheddo, Missione senza se e senza ma. L'annuncio alle genti dal Concilio a Papa Francesco, Bologna 2013, 119-120.

38 RMi 1.

39 Cfr. RMi 2; P. Gheddo, Missione senza se e senza ma. L'annuncio alle genti dal Concilio a Papa Francesco..., 122.
} 
dicale secolarizzazione della vita, la rinascita del fondamentalismo nazionalistico e religioso, il ripiegamento sulla cultura laica, la mondanizzazione della Chiesa, la speranza intra-storica legata al primato dello sviluppo economico, scientifico, tecnologico, biogenetico ecc. Invece tra i fattori interni si potrebbero annoverare: la considerazione teologica delle religioni come fonti della rivelazione e come vie della salvezza, e quindi un radicale pluralismo religioso, la sottovalutazione della vocazione missionaria della Chiesa, l'idea della Chiesa considerata e ridotta all'humanum inteso nella sua realizzazione orizzontale, immanente, sociale e del benessere umano (economico, politico, culturale, progresso tecno-scientifico ecc.), in fin dei conti una vaga e priva del significato più profondo 'auto-realizzazione della persona'.

Se il papa nella Redemptoris missio punterà e riprenderà gli elementi costitutivi del messaggio evangelico come la centralità di Cristo, la salvezza realizzata pienamente nel Regno di Dio inteso escatologicamente (senza sottovalutare la sua dimensione storica), la necessità della Chiesa, la presenza e attività universale dello Spirito Santo, l'annuncio della salvezza eterna a tutti i popoli, sarà perché tiene conto degli attuali e, radicalmente presenti nella vita della società globalizzata d'oggi, fenomeni particolari che esprimono la profonda crisi della fede. La civiltà post-moderna prima di tutto si caratterizza per il rifiuto di Dio: la cultura benché non neghi evidentemente l'esistenza di Dio, non lo ritiene più di alcun significato. Secolarizzazione, laicismo e ateismo pratico significano il rifiuto del rapporto con Dio e della presenza di Dio nella società e nella cultura moderna. Semmai la relazione con Dio assume una forma del tutto individualistica e ricondotta al piano privato, diventando una religione fai da te o credi a modo tuo. Sul piano epistemologico ed etico il primato viene dato all'effimero, come dirà il papa otto anni dopo nella Fides et ratio ${ }^{40}$. Per il relativismo non esiste una verità assoluta sull'uomo e sul suo destino, anzi è lo stesso relativismo che in certo qual modo diviene la nuova religione dell'uomo moderno ${ }^{41}$. Questi presupposti inevitabilmente portano, sul piano esistenziale, al nichilismo, cioè alla perdita del senso della vita. Se non esiste più una verità assoluta, non esistono più valori universali e meta-storici, quindi nulla per cui valga la pena di spendere la vita, difendere la verità anche dando la propria vita; tutto diventa provvisorio, transitorio, passeggero. Non si capisce più a che serve la vita dell'uomo, se non ad andare verso il nulla, cioè la morte, senza alcuna speranza ${ }^{42}$. Già da decenni nella cultura occidentale si può constatare la carenza della fede, l'eclissi del senso di Dio e dell'uomo,

40 Cfr. FR 46.

${ }^{41}$ Cfr. J. Ratzinger, Fede, verità, tolleranza, Siena 2003, 75, 87.

42 Cfr. P. Gheddo, Missione senza se e senza ma. L'annuncio alle genti dal Concilio a Papa Francesco..., 153-154. 
la mancanza di conoscenza reale della dottrina di Gesù Cristo, la presa di distanza di certi paesi dalle loro radici cristiane e anche quella che Giovanni Paolo II chiamava un'"apostasia silenziosa"43. Per questo i fenomeni specifici della cultura occidentale, caratterizzata dal 'proselitismo ateo', hanno portato Giovanni Paolo II a lanciare l'appello per una nuova evangelizzazione. L'immensa influenza economica, militare, tecnica, biogenetica e mediatica di un Occidente senza Dio potrebbe tradursi in un disastro per il mondo. Il cardinale Sarah, riallacciandosi all'appello di Giovanni Paolo II, afferma che se l'Occidente non si converte a Cristo e non risponde alla 'nuova evangelizzazione' potrebbe finire per paganizzare il mondo intero ${ }^{44}$.

A tale crisi è legato l'orientamento di colmare il vuoto esistenziale con le speranze intra-storiche che vedono la salvezza umana realizzata nelle dimensioni immanenti. Infatti "salvezza" è un termine con molti significati. C'è nella nostra epoca una tendenza generale a ridurla a qualcosa di puramente secolare e temporale ${ }^{45}$. "La tentazione oggi è ridurre il cristianesimo ad una sapienza meramente umana, quasi scienza del buon vivere. In un mondo fortemente secolarizzato è avvenuta una "graduale secolarizzazione della salvezza", per cui ci si batte, sì, per l'uomo, ma per un uomo dimezzato, ridotto alla sola dimensione orizzontale"46. Da qui nascono gli atteggiamenti comuni come materialismo e consumismo che vedono la vita umana volta ai beni terreni: benessere economico, potere politico, fama mediatica o carriera professionale. Già nel suo discorso ai partecipanti al congresso internazionale su "La salvezza oggi" tenutosi ad ottobre 1988 alla Pontificia Università Urbaniana, Giovanni Paolo II ha detto:

Correnti di pensiero e modelli di vita secolarizzati, sconvolgenti trasformazioni socio culturali, meravigliose conquiste della scienza e della tecnica, sollecitazioni pressanti dei mezzi di comunicazione, l'idea stessa o il pregiudizio circa tutto quello che costituisce la modernità, possono far credere all'uomo d'oggi che il concetto di salvezza

43 Un esempio dello "sradicamento" dalle origini cristiane si vede in certe posizioni delle Chiese dell'area germanica. In questo contesto può sembrare strano il silenzio di papa Francesco nel suo discorso ai vescovi tedeschi ricevuti il 20 novembre 2015 in cui ammonisce per certe tendenze nella Chiesa tedesca o indica i vari particolari della sua vita e, allo stesso tempo, non fa nessun accenno alla pratica estremamente diffusa e illegittima tra i pastori in Germania di ammettere i divorziati risposati alla Comunione Eucaristica. Cfr. I. Scaramuzzi, Strutture nuove e perfette non rispondono all'erosione della fede, http://vaticaninsider.lastampa.it/vaticano/dettaglio-articolo/articolo/francesco-francis-francisco-44783/.

44 Cfr. R. Sarah, Dio o niente. Conversazione sulla fede con Nicolas Diat..., 188-189.

45 Cfr. S. Karotemprel, Motivazioni e validità permanente della missione cristiana, in: AA.Vv., Riflessioni sulla "Redemptoris missio"..., 41-42.

46 RMi 11. 
si riduca all'ambito temporale, così che egli possa trovare in sé e nel suo progresso terreno quanto occorre per realizzarla ${ }^{47}$.

In tal modo si è realizzata la profezia articolata nella sua prima enciclica: "L'uomo d'oggi sembra essere sempre più minacciato da ciò che produce [...]; i frutti del suo lavoro si rivolgono contro l'uomo stesso [...] che pertanto vive sempre più nella paura" ${ }^{4}$. Si parla di una promozione umana e degli umanesimi laici che in effetti risultano incapaci di rispondere e appagare i più profondi aneliti del cuore umano. Senza ignorare i problemi e la condizione di estrema povertà materiale e lo sfruttamento subito da molte popolazioni del mondo (problema che è stato profondamente affrontato nelle encicliche sociali), il papa afferma: "Il contributo della Chiesa e della sua opera evangelizzatrice per lo sviluppo dei popoli riguarda non soltanto il Sud del mondo, per combattervi la miseria materiale e il sottosviluppo, ma anche il Nord, che è esposto alla miseria morale e spirituale causata dal super-sviluppo"49. Per tale motivo l'enciclica parlando dei destinatari e degli immensi areopaghi della missione cristiana contemporanea considera anche il mondo scristianizzato, ossia i popoli e le nazioni che avendo la loro storia e il loro passato strettamente legato alla fede cristiana comunque si sono sradicate da essi. In tale contesto il documento del magistero mette in guardia da qualsiasi concezione 'regnocentrica' della salvezza intesa o ridotta alla sua dimensione immanente, che pretenderebbe la realizzazione della missione della Chiesa in quanto impegnata primariamente o semplicemente nelle questioni sociali, politiche, economiche e così via. In questo contesto si capisce ancora di più il desiderio ardente di Giovanni Paolo II di ridare slancio alla vita missionaria della Chiesa, da lui chiamata nuova evangelizzazione in tempi in cui sempre più evidenti sono i disaccordi, i dissensi, le divisioni sulle questioni dogmatiche, etiche, legislative, pastorali e sociali non solo nel mondo, ma che penetrano anche la Chiesa provocando profondi disorientamenti tra i fedeli $i^{50}$.

Un'altra sfida di fronte a cui sta oggi la validità della missione evangelizzatrice della Chiesa è piuttosto di carattere interno e teologico; precisamente si tratta della sempre più diffusa teologia del pluralismo religioso che non di rado assume una forma radicale. Infatti un notevole indebolimento dello slancio missionario

47 http://www.fjp2.com/fr/jean-paul-ii/bibliotheque-en-ligne/discours/10585-to-the-participants-at-the-international-missiology-congress-held-by-the-urbanian-pontifical-university-october-7-1988.

48 RH 15.

49 RMi 59.

50 Cfr. R. Sarah, Dio o niente. Conversazione sulla fede con Nicolas Diat, 125, 149, 187-188. 
è anche dovuto in gran parte ad un certo relativismo religioso ${ }^{51}$ espresso da alcune correnti teologiche indicate dal $\mathrm{papa}^{52}$. Nella seconda metà del XX secolo la ricerca teologica su una piattaforma comune per l'umanità intera in ordine alla salvezza, ha portato molti teologi a optare per un'accettazione teoretica del pluralismo religioso. In modo molto sintetico, secondo la loro tesi, la salvezza e la rivelazione per l'uomo, sia che gli sia donata da un Dio personale, sia che provenga da forze intrinseche all'uomo stesso, si basa sull'evidenza della volontà salvifica universale di Dio confermata dalle Scritture; si basa inoltre sul fatto della presenza di uomini santi e buoni in tutte le religioni ispirate dalle autentiche esperienze di Dio; ed infine sul fatto che lo Spirito di Dio è all'opera in mezzo a tutti i popoli e nell'universo intero sempre nello stesso modo e sempre con gli stessi effetti. Secondo il pluralismo religioso radicale qualunque cosa faccia da intermediario tra il Divino, l'Assoluto, il Trascendente, è sempre assoluto per la salvezza dell'uomo. A questo punto tutte le religioni, compreso il cristianesimo, sarebbero solo varianti culturali dell'esperienza umana o probabili esperienze mistiche del Trascendente ${ }^{53}$. Secondo i propugnatori della teologia pluralista delle religioni la tradizionale pretesa cristiana sull'unicità e universalità della rivelazione salvifica in Gesù Cristo, sarebbe il risultato di condizionamenti culturali come tutte le altre esperienze religiose, e pertanto devono essere abbandonate in favore dell'unità umana, del reciproco rispetto, del dialogo svolto su una comune piattaforma ${ }^{54}$. Questi presupposti comportano alcune conseguenze sconcertanti per la comprensione teologica della missione cristiana: la proclamazione di Gesù Cristo come unico e universale mediatore della salvezza è "assurda", "arrogante", "mal interpretata", o almeno qualcosa da passare sotto silenzio nell'epoca del dialogo, della tolleranza e del mutuo arricchimento. La fede nell'unicità e universalità di Cristo in ordine alla salvezza è un'esperienza soggettiva senza una base obiettiva nella realtà e nella storia ${ }^{55}$. Tale impostazione teologica ha messo ovviamente in crisi la cristologia, l'ecclesiologia e la teologia della missione. Sono fallite le ragioni d'essere dell'attività missionaria dei cristiani. Per questo sembrano un grido di allarme le parole del cardinale Joseph Tomko, prefetto

${ }_{51}$ Cfr. A. Wolanin, Linee attuali della "theologia missionis", in: AA.Vv., Cristo Chiesa Missione. Commento alla "Redemptoris missio", Pontificia Università Urbaniana, Roma 1992, 42.

52 Cfr. RMi 35.

53 Cfr. W.C. Smith, Theology and the Worlds'Religious History, in: Towards a Universal Theology of Religion, ed. L. Swidler, New York 1978, 51-72; G. Kaufman, Religious Diversity, Historical Consciousness and Christian Theology, in: The Myth of Christian Uniqueness, eds. P. Knitter, J. Hick, New York 1988, 3-15; P. Knitter, No Other Name?, New York 1985.

54 G. Kaufman, Religious Diversity, Historical Consciousness and Christian Theology..., 3-15.

55 Cfr. P. Knitter, Hans Küng's Theological Rubicon, in: Towards a Universal Theology of Religion..., 225-229; L. Newbigin, The Gospel in a Pluralist Society, Grand Rapids 1989, 25. 
di Propaganda Fide, pronunciate nella prolusione al congresso internazionale, già menzionato prima, su "La salvezza oggi", tenutosi nel 1988 alla Pontificia Università Urbaniana di Roma che esponevano i dubbi e le obiezioni degli stessi missionari che provavano ed avvertivano come una ferita alla loro vocazione e all'impegno di tutta la vita: "che senso ha annunziare Cristo, se gli uomini si salvano anche senza di lui? Se la rivelazione cristiana è una delle tante espressioni della volontà di Dio? Se le altre religioni possono ugualmente portare a Dio e il dialogo può sostituire l'annuncio? Se l'evangelizzazione consiste principalmente nella promozione della giustizia sociale? Se i missionari non sono più accettati o graditi come annunciatori del Vangelo? Questa preoccupazione è stata espressa dal papa già all'inizio dell'enciclica ${ }^{56} \mathrm{e}$ in seguito ribadita in modo ancora più deciso:

una delle ragioni più gravi dello scarso interesse per l'impegno missionario è la mentalità indifferentista, largamente diffusa, purtroppo, anche tra i cristiani, spesso radicata in visioni teologiche non corrette e improntata a un relativismo religioso che porta a ritenere che «una religione vale l'altra» ${ }^{57}$.

Questo contesto storico - teologico è indispensabile per comprendere fino in fondo l'intenzione del papa di scrivere l'enciclica correggendo le idee sbagliate e chiarendo i fondamenti della fede e della missione, intrinsecamente legati. Innanzitutto ha come uno dei principali obiettivi quello di chiarire la confusione teologica sorta intorno alla missione alle genti, al dialogo con le religioni non cristiane e al rapporto fra l'annuncio di Cristo e lo sviluppo dell'uomo e dei popoli ${ }^{58}$. In tale contesto emerge ancor più la necessità dell'enciclica missionaria che con la sua esortazione e incoraggiamento, malgrado le difficoltà interne ed esterne, chiama a vincere i comportamenti pessimisti o inattivi: "Ciò che conta [...] è la fiducia che viene dalla fede, cioè dalla certezza che non siamo noi protagonisti della missione, ma Gesù Cristo e il suo Spirito" 59 . La Redemptoris missio è stata giudicata da alcuni teologi l'enciclica più rappresentativa del pontificato di Giovanni Paolo II, che con tutti i suoi viaggi, fino agli estremi confini del mondo, dava nitidamente l'idea di essere il Pontefice della Chiesa cattolica, cioè universale, missionaria. Diversi ne hanno lodato lo stile semplice e immediato. Il cardinale Godfried Daneels di Bruxelles ha scritto che è "il programma di lavoro per il prossimo millennio". Il cardinale Joseph Tomko, aveva ottenuto un'enciclica per il XXV dell'Ad gentes, l'unico fra i 16

$56 \quad$ Cfr. RMi 4.

57 RMi 36.

58 Cfr. P. Gheddo, Missione senza se e senza ma. L'annuncio alle genti dal Concilio a Papa Francesco..., 127.

59 RMi 36. 
documenti del Vaticano II aggiornato con un'enciclica. Tuttavia l'idea ricorrente a quel tempo, nelle alte sfere della Curia romana, era che un'enciclica per le missioni era troppo: non è più il momento di porre in risalto l'urgenza e il valore specifico della missione alle genti, poiché tutta la Chiesa è missionaria e tutti i popoli hanno bisogno di missione. Anche nell'opinione pubblica occidentale (e cattolica) l'enciclica ha avuto uno scarso impatto. L'enciclica missionaria non ha quasi fatto notizia e anche in seguito, la stampa cattolica e missionaria l'hanno quasi dimenticata, forse perché, secondo i primi affrettati commenti, non diceva nulla di nuovo rispetto all'Ad Gentes del Concilio Vaticano II. Anche parecchio tempo dopo, riviste teologiche cattoliche scrivevano che era una rilettura del Decreto conciliare.

Questa opinione rispecchia l'ignoranza anche da parte di chi l'ha letta, ma non l'ha capita in profondità. Prima di tutto l'enciclica ha confermato l'Ad gentes, in un momento in cui difficoltà esterne e interne, come è stato detto sopra, avevano indebolito lo slancio missionario della Chiesa. Il fatto che il papa abbia voluto fare un'enciclica specifica sul primo annunzio del Vangelo ai non cristiani, ha un significato importante che va richiamato! Anzi nell'enciclica dice: "Proprio il contatto diretto con i popoli che ignorano Cristo, mi ha ancor più convinto dell'urgenza di tale attività (missionaria)" ${ }^{\prime 60}$.

Giovanni Paolo II introduce anche l'espressione "nuova evangelizzazione", cioè nuova nel suo ardore, nei suoi metodi, nelle sue espressioni e anche in quanto rivolta al mondo scristianizzato ${ }^{61}$. Questo concetto, tanto caro a papa Wojtyła, è stato usato da lui stesso per la prima volta nel 1983 ad Haiti, in occasione dell'inizio del novenario per la celebrazione del $\mathrm{V}^{\circ}$ centenario dell'evangelizzazione nelle Americhe ${ }^{62}$.

\section{LA CHIESA MISSIONARIA DI FRONTE ALLE PROBLEMATICHE SFIDE DEL SINODO AMAZZONICO}

Solo brevemente e in modo sintetico ci si può soffermare ad individuare alcuni elementi prospettati, discussi o avvenuti durante il Sinodo Amazzonico (6-27 ottobre 2019, Roma) e riflettere su di essi alla luce della dottrina sulla Chiesa che come ricorda e ribadisce la Redemptoris missio - per sua natura è missionaria ${ }^{63}$. Una attenta lettura del documento finale del Sinodo, ma anche dell'Instrumen-

\footnotetext{
60 RMi 1.

${ }^{61}$ Cfr. G. Gutierréz, G.L. Müller, Dalla parte dei poveri. Teologia della liberazione, teologia della Chiesa, Padova 2013, 15, 42.

62 Cfr. M. Zago, Il contenuto della nuova evangelizzazione, in: Omnis Terra, aprile-giugno 1990, 104-109.

63 Cfr. RMi 5.
} 
tum laboris, suscita l'impressione di una certa discontinuità tra le linee principali dell'attuale magistero ecclesial ${ }^{64} \mathrm{e}$ il contenuto della Redemptoris missio è il suo linguaggio. Già due cose colpiscono in riferimento sia al sinodo stesso che al suo linguaggio. La prima cosa riguarda la stessa scelta di concentrarsi sulla regione amazzonica, come ragione "teologica"(?) per prendere in considerazione e analizzare alcune questioni della Chiesa nella sua universalità, ossia cattolicità. Il grosso rischio sta nel fatto che le soluzioni di carattere dottrinale, morale, disciplinare, pastorali e così via, derivanti dall'analisi della situazione particolare della Chiesa amazzonica possano avere ripercussioni inaccettabili per la Chiesa universale. La frammentazione della Chiesa percepibile attraverso il termine spesso usato "la Chiesa dal volto amazzonico" non ha molto a che fare con la Chiesa pluriforme di cui parla ad esempio Giovanni Paolo II nella Novo millennio ineunte ${ }^{65}$, a cui si richiama l'Instrumentum laboris del Sinodo, nel numero 110. Pare dunque che non sia metodologicamente giustificabile affinché i temi ecclesiologici inerenti la vita di tutta la Chiesa siano trattati secondo il detto "pars pro toto", con il ripiego alla fraintesa "pluriformità". Il secondo problema è relativo al linguaggio sinodale dove - riportando solo il testo del documento finale del Sinodo ${ }^{66}$ - non appaiono nemmeno una volta tra i concetti specifici della fede della Chiesa: rivelazione, salvezza, redenzione, risurrezione, eternità, penitenza, santità (santo), Santissimo Sacramento, Trinità, adorazione ecc. Ed altri, come preghiera, liturgia, peccato (nel senso strettamente teologico) - appaiono una sola volta. Invece 29 volte sono citati i termini: cultura, inculturazione, culturale, interculturalità. Il sinodo dimostra evidentemente il suo orientamento orizzontale con gli accenti posti sulle questioni sociali, ambientaliste, ecologiche, politiche, pluralistiche (teologia delle religioni), perdendo di vista, allo stesso tempo, ciò che fa parte della sua identità e della sua natura.

\section{LA STRATEGIA DEL SINODO: COSA E COME FARE PER CAMBIARE LA DOTTRINA DI FEDE E DI MORALE?}

La prima questione che si impone riguarda la domanda: si può essere sicuri che al sinodo a Roma conoscano la regione di cui si stanno occupando in questi giorni? Non è forse che l'Amazzonia di cui si parla è solo un luogo teologico

${ }_{64}$ Anche se il sinodo non ha di per sé il valore del magistero ufficiale, ma ha un notevole influsso su di esso.

65 Cfr. Giovanni Paolo II, Lettera apostolica, "Novo millennio ineunte" 40, 6 gennaio 2001, http:// w2.vatican.va/content/john-paul-ii/it/apost_letters/2001/documents/hf_jp-ii_apl_20010106_ novo-millennio-ineunte.html.

66 Sinodo Amazonico, Il Documento finale: Chiesa alleata dell'Amazzonia, https://www.vaticannews.va/it/vaticano/news/2019-10/sintesi-documento-sinodo-chiesa-alleata-amazzonia.html. 
preso in ostaggio da un certo modo di fare missione con lo scopo di stravolgere la dottrina e introdurre una nuova fede ${ }^{67}$. Infatti 1'Instrumentum laboris ${ }^{68}$ sembra dimostrare una insufficiente e unilaterale conoscenza ed esposizione della vera realtà di tutta la zona amazzonica. Davvero l'intera popolazione amazzonica è da considerare nativa? Almeno non in Venezuela. Nelle diocesi già stabilite in questa regione, non nei vicariati, vi è una maggioranza di creoli, venezuelani bianchi o misti e afro-venezuelani che non hanno quella cultura indigena. Lo stesso accade a Manaus e Belem, in Brasile ${ }^{69}$.

Secondo il vescovo Robert Mutsaerts, ausiliare di Hertogenbosch in Olanda, la Chiesa durante questo Sinodo per l'Amazzonia parla di qualcosa di diverso da ciò che effettivamente vuole raggiungere ${ }^{70}$. Tra il parlare (dialogare, discutere, incontrarsi, condividere ecc.) e il fine del sinodo c'è un abisso quanto ai contenuti da raggiungere. Ogni volta si sentiva suonare la stessa melodia: nuovi percorsi, l'ascolto delle popolazioni indigene, il cambiamento climatico e la Madre Terra. Sembra che nessuno voglia veramente menzionare i problemi fondamentali ${ }^{71}$.

In simile modo si esprime il cardinale venezuelano Jorge Urosa Savino:

è sorprendente che la maggior parte dei commenti sul Sinodo, fatti di recente dagli ecclesiastici, siano collegati alla sua preparazione, toccando solo o principalmente l'aspetto ecologico e i problemi di ordine sociale ed economico delle popolazioni amazzoniche. Questa sembra essere la cosa più importante per la Chiesa. Toccano poco l'aspetto religioso e spirituale della missione della Chiesa di annunciare la Parola e comunicare i doni di Cristo all'umanità. Anche il documento di lavoro dà questa

67 Cfr. A. Zambrano, "In quei riti c'è il diavolo". Parola di vescovo amazzonico, 23.10.2019, https://lanuovabq.it/it/in-quei-riti-ce-il-diavolo-parola-di-vescovo-amazzonico.

68 Assemblea speciale del Sinodo dei vescovi per la regione panamazzonica, Instrumentum laboris, "Amazzonia: nuovo cammini per la Chiesa per la una ecologia integrale", Vaticano, 17 giugno 2019, http://www.sinodoamazonico.va/content/sinodoamazonico/it/documenti/l-instrumentum-laboris-per-il-sinodo-sull-amazzonia1.html.

69 Cfr. J. Urosa Savino, Amazzonia, quando parliamo di evangelizzazione?, 1.10.2019, https:// lanuovabq.it/it/amazzonia-quando-parliamo-di-evangelizzazione.

70 Ad esempio quanto al problema degli indios in Amazzonia. L'Amazonia non sta per essere distrutta, l'Amazzonia è intatta. Non ha fondamento il mito dell'Amazzonia che brucia. [...] Secondo un recente studio dell'Istituto brasiliano di geografia e statistiche gli indios nel Paese sarebbero 897mila, di cui solo 180mila vivono in Amazzonia. Come si giustifica un Sinodo di fronte a un numero tanto ridotto di popolazione? Cfr. Il sinodo per l'Amazzonia, forse una nuova religione tribale?, https://cronicasdepapafrancisco.com/2019/10/08/il-sinodo-per-lamazzonia-forse-una-nuova-religione-tribale/.

71 Cfr. A. Zambrano, Il Sinodo per l'Amazzonia segue un'agenda politicamente corretta, 24.10.2019, https://anticattocomunismo.wordpress.com/2019/10/24/lagenda-politicamente-corretta-del-sinodo-sullamazzonia/. 
impressione, quindi nel Sinodo si dovrà correggere il percorso e si dovrà mettere in evidenza la centralità dell'azione evangelizzatrice e pastorale per la rivitalizzazione della Chiesa in 'Amazzonia ${ }^{72}$.

Di fronte a tale preoccupante domanda non si sottrae il cardinale Müller spiegando schiettamente che oggi è "assai inquietante come in alcuni «testi ecclesiastici di riforma» non venga fatta alcuna menzione di Dio, di Cristo, delle Sacre Scritture dell'Antico e Nuovo Testamento, o come il Vangelo rischi di venir soffocato sotto un mucchio di retorica relativa alla preoccupazione socio-psicologica e pastorale" 73 . E poi aggiunge che i cattolici non credono in

un Dio pagano che ci parla nei miti e nelle utopie, nella dinamica degli eventi, nei processi da noi avviati, nel sangue della razza, nello spirito popolare o nelle realtà immorali della vita. La teologia riconosce come locus theologicus soltanto l'unica Parola di Dio nella Sacra Scrittura e nella Tradizione, mentre il Magistero può solo rivendicare un'autorità interpretativa ${ }^{74}$.

Proprio come gli apostoli, anche i vescovi e i sacerdoti sono solo ed esclusivamente "servi di Cristo e amministratori dei misteri di Dio ai quali si richiede solo che ognuno risulti fedele". Non devono reinventare il cristianesimo, considerando se stessi più saggi di Gesù stesso, che sarebbe ancora limitato dalla vecchia visione del mondo e i cui insegnamenti avrebbero urgente bisogno di essere adattati al pensiero illuminato dei suoi discepoli di oggi $i^{75}$.

Sulla linea del ragionamento del cardinale Müller, anche Martin Hähnel dell'Università Cattolica di Eichstätt-Ingolstadt asserisce che Il Sinodo sembra essere il punto focale di un' "agenda ecclesiale". La Chiesa moderna, soprattutto dal XIX sec., ha sostituito l'idea della Divina Provvidenza con il progresso; sostituzione che è stata accompagnata dalla liquefazione della dogmatica e dalla relativizzazione delle norme morali. Questo processo sta raggiun-

72 J. Urosa Savino, Amazzonia, quando parliamo di evangelizzazione?, 1.10.2019, https://lanuovabq.it/it/amazzonia-quando-parliamo-di-evangelizzazione.

$73 \mathrm{Cfr}$. M. Tosatti, Papa e vescovi servono il Magistero, non ricevono nuove rivelazioni neopagane, 28.09.2019, https://lanuovabq.it/it/papa-e-vescovi-servono-il-magistero-non-ricevono-nuove-rivelazioni-neopagane.

74 M. Tosatti, Papa e vescovi servono il Magistero, non ricevono nuove rivelazioni neopagane, 28.09.2019, https://lanuovabq.it/it/papa-e-vescovi-servono-il-magistero-non-ricevono-nuove-rivelazioni-neopagane.

$75 \mathrm{Cfr}$. M. Tosatti, Papa e vescovi servono il Magistero, non ricevono nuove rivelazioni neopagane, 28.09.2019, https://lanuovabq.it/it/papa-e-vescovi-servono-il-magistero-non-ricevono-nuove-rivelazioni-neopagane. 
gendo il suo vertice proprio nel Sinodo e ne è segno il fatto che l'assise sinodale si mostra priva della forza di forgiare una cultura ed una mentalità. Non è più il cristianesimo che ha la forza di "incorporare" il mondo, come, per esempio, San Tommaso seppe "battezzare" Aristotele, ma il contrario. Questo adeguamento della Chiesa allo spirito del mondo è la ragione per cui il mondo riesce ad imporre le sue esigenze, alle quali la Chiesa sta cedendo sempre di più. Hähnel ricorda che nel 2011 a Friburgo, in occasione del suo viaggio apostolico in Germania, Benedetto XVI aveva cercato di fermare questo processo, chiedendo alla Chiesa di "de-mondanizzarsi" ".

Secondo l'opinione di A. Strumia, la crescente tendenza ad attenuare l'importanza della dottrina e della verità all'interno della Chiesa è un fenomeno palese ed esplicito, quasi di moda, nella prassi del mondo cattolico. La sua causa secondo alcuni risiede nel rifiuto del fondamento metafisico della teologia, nell'assunzione di una teologia "narrativa" poggiata su esperienze ed opinioni soggettive. Ora da qualche tempo, in particolare con il pontificato di papa Francesco, ci si chiede di trovare il modo per eliminare il "fondamento" metafisico della teologia e quindi, di conseguenza, per cambiare la dottrina di fede e di morale. Il metodo assunto consiste nel puntare sugli aspetti storico-esperienziali che aprono le porte alle ideologie come ambientalismo, animalismo, ecologismo, pauperismo, immigrazionismo illimitato e in questi ultimi giorni al panteismo e al paganesimo che hanno fatto ingresso anche in Vaticano, non limitandosi a fermarsi sulla facciata della basilica di san Pietro ${ }^{77}$.

\section{TRA L'INCULTURAZIONE CRISTIANA E LA PAGANIZZAZIONE DEL CRISTIANESIMO}

Già l'Instrumentum laboris del sinodo parlava dell'inculturazione della fede, ma dando un valore quasi autonomo e autosufficiente alle culture native in ordine alla salvezza e non valorizzando o proponendo l'evangelizzazione dei popoli anche attraverso l'inculturazione. Questa infatti è la necessaria trasformazione dell'esistenza umana e della vita religiosa, sociale, culturale e familiare dei popoli attraverso il Vangelo di Cristo e la morale biblica, cristiana e cattolica ${ }^{78}$. Nell'ottica della Redemptoris missio l'incarnazione dell'Evangelo nelle tradizioni dei

76 Cfr. L. Scrosati, Chiesa mondana e senza verità: come siamo arrivati fino a qui, 27.10.2019, https://lanuovabq.it/it/chiesa-mondana-e-senza-verita-come-siamo-arrivati-fino-a-qui.

77 Cfr. A. Strumia, Come ha fatto la Chiesa a ridursi così?, 11.10.2019, https://anticattocomunismo.wordpress.com/2019/10/11/come-ha-fatto-la-chiesa-a-ridursi-cosi/.

78 Cfr. A.A. Roest Crollius, Missione e inculturazione, in: La missione del Redentore. Studi sull'enciclica missionaria di Giovanni Paolo II, eds. E. dal Covolo, A. Triacca,Torino 1992, 247-255. 
popoli avviene mediante l'inculturazione ${ }^{79}$, cioè l'intima "trasformazione degli autentici valori culturali mediante l'integrazione nel cristianesimo e il radicamento del cristianesimo nelle varie culture" ${ }^{80}$. È un punto che deve essere rivisto e migliorato ${ }^{81}$.

Una delle più grandi preoccupazioni dei fedeli che seguivano le voci dell'aula sinodale e gli eventi che accompagnavano il sinodo, erano i riti tribali delle varie tradizioni religiose, che avendo il carattere pagano, entravano nelle cerimonie svolte nelle chiese cattoliche e proprio lì, in presenza perfino dei partecipanti al sinodo, entravano come parte integrante della celebrazione liturgica o para-liturgica. Questi riti pagano-amazzonici si sono celebrati in questi giorni perfino in Vaticano, a cominciare dalla cerimonia nei Giardini Vaticani sotto lo sguardo del Papa. Il Sinodo sull'Amazzonia entrerà nella storia come il Sinodo di Pachamama? Chi è Pachamama? È la Dea terra, una divinità pagana venerata dai Padri Sinodali riuniti a Roma in queste settimane. L'immagine di Pachamama, ha fatto la sua apparizione nei Giardini Vaticani, il 4 ottobre (festa di san Francesco d'Assisi), alla vigilia dell'apertura del Sinodo sull'Amazzonia. Nel corso di una cerimonia guidata da una donna india dell'Amazzonia, alla presenza di papa Francesco e di cardinali e vescovi, sono state adorate due statuette in legno di Pachamama, raffiguranti due donne nude e incinte una di fronte a un'altra, mentre un'altra statuetta rappresentava un soggetto maschile nudo e pronto all'atto sessuale.

L'8 ottobre la statua di Pachamama è ricomparsa nella chiesa di Santa Maria in Traspontina, prima in un cesto, poi in una canoa trasportata nella Chiesa nel corso di un'altra cerimonia pagana organizzata dalla Rete Ecclesiale Panamazzonica (REPAM). In una cappella laterale di Santa Maria in Traspontina è stato sistemato un poster per mostrare che "tutto è collegato", secondo l'insegnamento della Laudato sì di papa Francesco. In esso si vede la foto di un animale, un piccolo mammifero che succhia il seno di una donna indigena nuda che sull'altro braccio porta un bambino. Infine la Pachamama, sempre sulla sua canoa, è ricomparsa nella Via Crucis amazzonica, (secondo tanti fedeli una cosa blasfema), che si è svolta il 19 ottobre alla presenza, tra gli altri, del cardinale Pedro Barreto, vicepresidente della REPAM, che ha organizzato tutti questi eventi che difficilmente possono essere visti come vera inculturazione secondo il pensiero il Concilio Vaticano II e il magistero successivo, inclusa soprattutto la Redemptoris missio. Piuttosto si potrebbe parlare di una precisa e deliberata manovra di infiltrare all'interno della fede della Chiesa elementi che le sono intrinsecamente contraddittori.

79 Cfr. RMi 52-54.

80 RMi 52.

81 J. Urosa Savino, Amazzonia, quando parliamo di evangelizzazione?, 1.10.2019, https://lanuovabq.it/it/amazzonia-quando-parliamo-di-evangelizzazione. 
Fino al 21 ottobre, le statuette di Pachamama sono state ospitate nella chiesa della Traspontina, dove ogni giorno si è svolto un rituale amazzonico di carattere magico ed esoterico, cioè diabolico, intitolato "Momenti di spiritualità amazzonica" $"$.

La cosiddetta Chiesa dal volto amazzonico si presenta piuttosto come un prodotto da laboratorio, frutto delle direttive dell'Instrumentum laboris, più che un esito inculturato di un seme cristiano che, immesso nella cultura primigenia, ha portato a un culto e a una cultura cattolica. Il 12 ottobre è stata celebrata la Misa por la Tierra Sin Males, creata nel 1979 e già condannata dalla Santa Sede. Veniva "celebrata" in Brasile nell'ambito della Teologia india e in occasione del Sinodo di nuovo riproposta nella chiesa di Santa Maria in Transpontina in un trionfo di rivendicazioni anticolonialiste e senza alcunché di cattolico. Anzi, si chiede perdono per il battesimo imposto agli Indios come un marchio impresso nel corpo e si loda la terra che è stata saccheggiata. Si tratta dunque di una messa blasfema in cui il battesimo viene bestemmiato e definito come il "marchio del bestiame umano" $" 83$ - spiega Josè Antonio Ureta ${ }^{84}$, studioso e autore del noto libro "Il «cambio di paradigma» di Papa Francesco: continuità o rottura nella missione della Chiesa?: Bilancio quinquennale del suo pontificato" 85 .

La risposta ai riti pagani di questi giorni a Roma arriva da un vescovo amazzonico che conosce l'Amazzonia più di molti padri sinodali. Di fatto, il vescovo emerito di Belem, Azcona, ha spiegato che in certi di 'quei riti c'è il diavolo, c'è la magia [...] Quelli visti in questi giorni sono sacrilegi demoniaci che producono scandalo. Noi in Amazzonia questo lo sappiamo bene"86. In seguito, riferendosi sempre alla statua di Pachamama, il vescovo Azcona ha ribadito che essa "non è e non sarà mai la Vergine Maria. Dire che quella statua rappresenta la Madonna è

82 Cfr. R. de Mattei, Alla fine il Sinodo sull'Amazzonia entrerà nella storia come il Sinodo di Pachamama, 25.10.2019, https://www.radioromalibera.org/cultura-cattolica/analisi-e-commenti/ il-sinodo-di-pacha-mama/.

83 Cfr. A. Zambrano, Hanno portato via il Signore! L'Amazzonia entra in chiesa con un culto pagano, 12.10.2019, https://lanuovabq.it/it/hanno-portato-via-il-signore-lamazzonia-entra-in-chiesa-con-un-culto-pagano.

84 Josè Antonio Ureta è membro dell'Associazione Tradizione Famiglia Proprietà (TFP), nata per diffondere il pensiero e l'azione di un grande leader cattolico, il prof. Plinio Corrêa de Oliveira (1908-1995), ispiratore di un'opera spirituale ormai diffusa in tutto il mondo. In Italia, la TFP pubblica il trimestrale Tradizione Famiglia Proprietà, e organizza conferenze ed incontri, collaborando a questo scopo con altre realtà del mondo cattolico, https://www.atfp.it/chi-siamo/108le-societa-per-la-difesa.

85 Cfr. J.A. Ureta, Il "cambio di paradigma" di Papa Francesco: continuità o rottura nella missione della Chiesa?: Bilancio quinquennale del suo pontificato, San Paolo 2018.

86 Cfr. A. Zambrano, "In quei riti c'è il diavolo". Parola di vescovo amazzonico, 23.10.2019, https://lanuovabq.it/it/in-quei-riti-ce-il-diavolo-parola-di-vescovo-amazzonico. 
una bugia. Non è la Signora dell'Amazzonia perché l'unica Signora dell'Amazzonia è Maria di Nazareth. Non facciamo mescolanze sincretiste. Tutto ciò è impossibile: la Madre di Dio è la Regina del Cielo e della terra" ${ }^{87}$. In tale contesto il vescovo Azcona riferendosi ai principi teologici e pastorali dell'Instrumentum laboris, sottolinea l'urgenza del discernimento dello Spirito Santo che tanto piace a parlare al papa Francesco. Bisogna dunque "distinguere quello che viene dal demonio o dalla mente umana da quello che viene dallo Spirito Santo. Questo discernimento è fondamentale oggi per appartenere alla Chiesa e molto più per evangelizzare" $"$.

\section{LA DE-FALSIFICAZIONE DEL TERMINE "PROSELITISMO" E LA PERMANENTE VALIDITÀ DEL MANDATO MISSIONARIO}

Nelle sue riflessioni il vescovo olandese Mutsaerts nota che "i missionari vengono improvvisamente ritratti come imperialisti che hanno imposto i loro valori ai popoli indigeni. Questo significa che questi missionari non hanno mai significato nulla per i popoli indigeni? Hanno rischiato la vita per proclamare il Vangelo. Quanti martiri ci sono ora?". Il fatto è che tutto questo non ha nientea che vedere con la compassione per le tribù indigene. Sta diventando sempre più chiaro che il Sinodo viene usato impropriamente per portare avanti un'agenda nascosta ${ }^{89}$.

Di fronte alla diversità delle religioni, culture, nazioni, popoli, tradizioni la Chiesa "nulla rigetta di quanto è vero e santo in queste religioni [...] Tuttavia essa annuncia, ed è tenuta ad annunciare, il Cristo che è «via, verità e vita»" ${ }^{\circ}$. In forza di questo compito, affidato alla Chiesa intera, ogni cristiano è chiamato alla "diffusione del regno di Cristo su tutta la terra a gloria di Dio Padre, [e a] rendere partecipi tutti gli uomini della salvezza operata dalla redenzione, e per mezzo di essi ordinare effettivamente il mondo intero a Cristo"91.

Il documento finale del Sinodo nel capitolo III in cui parla della "conversione culturale" esprime la preoccupazione, mettendo in guardia i cristiani e i missionari dal "proselitismo". Infatti dice:

87 Cfr. ibidem.

88 Cfr. ibidem.

89 Cfr. A. Zambrano, Il Sinodo per l'Amazzonia segue un'agenda politicamente corretta, 24.10.2019, https://anticattocomunismo.wordpress.com/2019/10/24/lagenda-politicamente-corretta-del-sinodo-sullamazzonia/.

90 Concilio Vaticano II, Dichiarazione sulle relazioni della chiesa con le religioni non-cristiane "Nostra aetate", 2, EV 1/853-871.

91 Concilio Vaticano II, Decreto sull'apostolato dei laici "Apostolicam actuositatem", 2, EV 1/912-1041. 
Nell'ottica, poi, dell'inculturazione - ovvero dell'incarnazione del Vangelo nelle culture indigene - spazio viene dato alla teologia india e alla pietà popolare, le cui espressioni vanno apprezzate, accompagnate, promosse e talvolta "purificate", poiché sono momenti privilegiati di evangelizzazione che devono condurre all'incontro con Cristo. L'annuncio del Vangelo, infatti, non è un processo di distruzione, ma di crescita e di consolidamento di quei semina Verbi presenti nelle culture. Di qui, il rifiuto netto di "un'evangelizzazione in stile colonialista" e del "proselitismo", in favore di un annuncio inculturato che promuova una Chiesa dal volto amazzonico, in pieno rispetto e parità con la storia, la cultura e lo stile di vita delle popolazioni locali ${ }^{92}$.

Sembra che questo passo del documento finale non c'entra nulla con l'attuale situazione in cui si trova la Chiesa, come se il ragionamento degli autori di questo brano fosse completamente staccato dalla realtà e dal contesto storico in cui vivono i cristiani. Come parlare oggi del rischio di "un'evangelizzazione in stile colonialista" se, prima di tutto, le questioni politiche dei paesi "colonizzanti" sono del tutto separate dalle questioni di religione, a nome del principio, comunemente assunto dal mondo moderno, cioè della separazione o dell'autonomia tra la politica (l'ordine laico) e la Chiesa (l'ordine della fede). In secondo luogo, come è possibile parlare del proselitismo oggi, quando il 25-30\% (300 milioni) delle popolazioni cattoliche vive nelle condizioni di persecuzione proprio per il fatto di essere cristiani o membri della Chiesa? Si dovrebbe chiedere ai padri sinodali in quale parte del mondo sarebbe così evidente l'agire distruttivo da parte della Chiesa nei confronti delle culture, tradizioni, credenze ecc.; da questo testo sinodale emerge una grave ignoranza quanto al significato del termine "proselitismo".

Il sinodo acriticamente e in maniera unisona con gli enunciati di papa Francesco ha assunto il concetto di "proselitismo" nella sua connotazione unilaterale e negativa. Spesso nei suoi discorsi usava il linguaggio tipo "il proselitismo è una solenne sciocchezza", "fare proselitismo nel campo ecclesiale è peccato" ed ancora "il proselitismo è un veleno per il cammino ecumenico". Limitandosi a queste espressioni sembra che il sinodo, seguendo fedelmente le idee del papa, è rimasto nel remoto passato, in alcuni periodi storici definitivamente tramontati e da parecchio tempo superati.

Invece nell'Enciclopedia Treccani, riguardo al termine "proselitismo" si legge che è una

attività svolta da una religione, un movimento, un partito per cercare e formare nuovi seguaci. Mentre le religioni tribali e nazionali non hanno alcuna tendenza a estender-

92 Sinodo Amazonico. Il Documento finale: Chiesa alleata dell'Amazzonia, https://www.vaticannews.va/it/vaticano/news/2019-10/sintesi-documento-sinodo-chiesa-alleata-amazzonia.html. 
si al di là della comunità sociale che le pratica, le religioni sopranazionali tendono a esercitare proselitismo, partendo dalla convinzione di essere vera religione, non legata a un singolo popolo, ma valida per tutti e di rappresentare la via di salvezza per ogni individuo umano: mossi da ideali soteriologici i rappresentanti della religione cercano di conquistare il maggior numero di proseliti. L'attività missionaria è una forma organizzata del proselitismo ${ }^{93}$.

L'Enciclopedia precisa poi che si tratta di una attività tesa a cercare i proseliti e formarli. In ogni modo che cosa c'è di male o di inaccettabile nel concetto di "proselitismo" per come lo definisce una laica enciclopedia? Applicando il riferimento enciclopedico alla Chiesa, la prima componente del "proselitismo" è la convinzione di "essere vera religione". Questa formula intende dire non tanto la superiorità sul piano meramente cognitivo, ma si riferisce alla Chiesa, realtà umano-divina, storico-trascendente, depositaria della Verità di natura soprannaturale, unita all'opera salvifica di tutta l'umanità. In altre parole si tratta della verità contenuta, formulata e trasmessa nel Credo, nell'evento dell'unica auto-comunicazione di Dio avvenuta nella storia universale con l'incarnazione del Figlio di Dio fattosi uomo. Se non ci fosse questa fede/convinzione, allora che ne sarebbe della Chiesa? A che cosa essa servirebbe nella storia dell'umanità, dato che era voluta de iure da Dio sin dall'eternità?

Altri due termini che spiegano il significato del "proselitismo" sono "cercare" e "formare". L'Enciclopedia non parla affatto di imposizione, costrizione, repressione, violenza, oppressione ecc. È una cosa naturale che, se la Chiesa è cosciente del mandato che le è stato affidato (Mt 28,18-20; Mc 16,15-20; Lc 24,46-53; Gv 17,17-19; 20,21-22), non può essere chiusa, ermetica, auto-referenziale, ma una "Chiesa in uscita", come ripete spesso papa Francesco. Ne deriva che la Chiesa si muove, va in cerca di chi è predisposto e interessato ad ascoltare e conoscere il suo messaggio. Una volta accettato il messaggio, un convertito o un proselita inizia una permanente formazione circa il nuovo stile di vita fondato sulla verità ascoltata, conosciuta, accolta e vissuta. L'Enciclopedia definisce il "proselitismo" come una forma organizzata dell'attività missionaria; nel caso specifico, dell'attività evangelizzatrice svolta dalla Chiesa sin dalle sue origini, che risalgono sia alla storia pre-pasquale di Gesù, che alla storia della comunità dei cristiani dopo la risurrezione di Cristo. È chiaro che il "proselitismo" inteso in questo senso implica sia la "attrazione", sia la "intrinseca bellezza del Vangelo", sia la "credibile testimonianza" di chi annuncia.

93 http://www.treccani.it/enciclopedia/proselitismo/. 


\section{FRONTE ALLA RIDUZIONE 'SOCIOLOGISTA' DELLA MISSIONE DELLA CHIESA}

Si discuteva dell'ambiente, dell'innalzamento del livello del mare di ripetere il mantra che occorre "ascoltare". I padri sinodali, secondo Mutsaerts, parlavano "come politici, usando gli stessi slogan, la stessa retorica da quattro soldi. È strano che in un sinodo questi argomenti debbano essere oggetto di discussione. Non è competenza della Chiesa, non è la nostra attività principale e non è la nostra

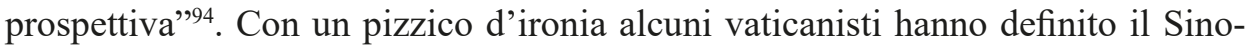
do come un corso accelerato di climatologia ${ }^{95}$. Il 22 ottobre al briefing c'erano ben due vescovi amazzonici e un cardinale congolese, che hanno parlato quasi esclusivamente di cambiamenti climatici e di catastrofi prossime venture se in Occidente non si cambiano subito gli stili di vita. A parte le sciocchezze che sono state dette - ma non si può pretendere diversamente, con un corso così accelerato è facile che idee e concetti si confondano - è stato interessante notare quello che è accaduto all'ultima domanda: dopo un'ora di catechismo ecologista, una persona ha fatto la domanda sull'evangelizzazione. Sono seguiti alcuni attimi di smarrimento. Evangelizzazione? Quale evangelizzazione? È stato come se alla riunione di un consiglio d'amministrazione di una grande multinazionale qualcuno si alzasse improvvisamente in piedi e dicesse: "Adesso recitiamo una preghiera". Poi il cardinale ha detto qualcosa, ma le facce viste al momento della domanda erano molto più significative della risposta ${ }^{96}$. Questo episodio che rispecchia tut-

94 Cfr. A. Zambrano, Il Sinodo per l'Amazzonia segue un'agenda politicamente corretta, 24.10.2019, https://anticattocomunismo.wordpress.com/2019/10/24/lagenda-politicamente-corretta-del-sinodo-sullamazzonia/.

95 A proposito dei cambiamenti climatici e pericoli di cui sarebbe minacciata l'Amazzonia, Luiz Carlos Molion dice che non esistono mutazioni climatiche o riscaldamento globale prodotti dagli uomini. Quella che esiste è una variazione naturale del clima. Ad esempio ci sono argomenti fisici concreti che suggeriscono che il riscaldamento globale tra il 1916 e il 1945 è stato causato dall'attività solare (la maggiore degli ultimi 400 anni). Oppure che il riscaldamento del 1976-2005, attribuito a attività umane, è invece stato causato dalla riduzione della copertura nuvolosa del $5 \%$ e dalla grande frequenza di eventi come El Niño. Lo stesso studioso smaschera il mito secondo cui l'Amazzonia sia polmone verde del mondo. In realtà l'Amazzonia non è essenziale per la distribuzione delle piogge in altre regioni lontane dall'America del Sud, perché l'Amazzonia non è fonte di umidità per l'atmosfera. La foresta consuma più ossigeno di quanto ne produca. La foresta non produce acqua, ricicla appena l'acqua di piogge anteriori. Cfr. Il sinodo per l'Amazzonia, forse una nuova religione tribale?, https://cronicasdepapafrancisco.com/2019/10/08/il-sinodo-per-lamazzonia-forse-una-nuova-religione-tribale/; cfr. anche Marinellys Tremamunno, L'Amazzonia non è il «polmone verde». Né un paradiso, 18.10.2019, https://lanuovabq.it/it/lamazzonia-non-e-il-polmone-verde-ne-un-paradiso.

96 Cfr. R. Cascioli, Da Pompili a Tornielli, due giorni di parole in libertà, 23.10.2019, https:// lanuovabq.it/it/da-pompili-a-tornielli-due-giorni-di-parole-in-liberta.

TwP 14,1 (2020) 
to il clima del sinodo e il suo procedimento, impone l'interrogativo sulla vera missione della Chiesa, su ciò che è da considerare il suo primo obiettivo ${ }^{97}$. La risposta sarà possibile solo se i pastori e le guide più responsabili nella Chiesa sapranno rispondere alla domanda: che cosa è la Chiesa? Quale è la sua natura e la sua identità? Anzi chi è la Chiesa ( $^{98}$. Ogni attività da parte della Chiesa, anche quella di carattere sociale, politico incluse le questioni ecologiche ecc., devono essere onticamente precedute da ciò che costituisce la missione della Chiesa, quella missione che appartiene alla sua natura:

E poiché la Chiesa è in Cristo come sacramento, cioè segno e strumento dell'intima unione con Dio e dell'unità di tutto il genere umano, il sinodo intende illustrarne con maggior chiarezza [...] questo dovere della Chiesa: uniti fra di loro dai vincoli sociali, economici e culturali, raggiungano anche la loro piena unità in Cristo $^{99}$.

Il primo compito affidato alla Chiesa è la proclamazione e l'annuncio del Vangelo della salvezza compiuta in Cristo. Il suo darsi da fare è, in primis e per sua natura, pensare alla salvezza di ogni persona umana, alla salvezza non nel senso orizzontale, temporaneo, storico-sociale del benessere materiale (senza ovviamente trascurarlo e ignorarlo), ma verticale, trascendente, in riferimento all'eternità e alla comunione di vita con Dio. Ecco la ragione per cui esiste nella storia dell'istituzione divina la Chiesa, orientata però verso la dimensione meta-storica e soprannaturale.

\section{L'ABBANDONO DELLA PASTORALE-VOCAZIONALE: 'LAICIZZAZIONE' DEL SACERDOZIO}

Secondo lo spirito conciliare sul piano missionario viene concesso maggior spazio ai laici e vengono a loro affidati molti nuovi impegni o funzioni, riservati prima solo ai ministri ordinati o alle persone consacrate. Tuttavia lo scarso interesse verso la pastorale vocazionale sembra essere abbastanza palese nel contesto dell'evangelizzazione e potrebbe falsamente indurre a pensare che ci possa essere

97 Cfr. L. Scrosati, Il Sinodo che ha dimenticato la salvezza delle anime, 28.10.2019, https://lanuovabq.it/it/il-sinodo-che-ha-dimenticato-la-salvezza-delle-anime.

98 Cfr. K. Parzych-Blakiewicz, M.A. Kopiec, "Sumienie eklezjalne" w blasku Prawdy, Dobra i zbawienia”, „Teologia w Polsce” 12 (2018) 2, 143-160.

99 Concilio Vaticano II, Costituzione dogmatica sulla chiesa "Lumen gentium”, 1, EV 1/284-456. 
come una sorta di "sostituzione" dei sacerdoti con i laici ${ }^{100}$. È interessante notare come il sinodo amazzonico voglia risolvere il problema della mancanza delle vocazioni sacerdotali, dei religiosi e dei missionari, usando i mezzi puramente pragmatici e mondani. Il tentativo sinodale mirato alla creazione di un clero non continente sembra essere fuori strada. L'idea di ordinare i viri probati sembra una deliberata scorciatoia per sottovalutare il sacramento dell'ordinazione e far apposta calare il numero delle vocazioni sacerdotali e missionarie, soprattutto quando continuamente e in ogni possibile occasione si parla del cosiddetto "clericalismo" come il male peggiore nella Chiesa?

Già nel 1990 san Giovanni Paolo II ha escluso ogni possibilità di intavolare una discussione all'interno della Chiesa in merito all'ordinazione dei viri probati $^{101}$. Vale la pena citare per intero il passaggio del discorso pronunciato dal pontefice polacco in occasione dell'VIII Assemblea generale ordinaria del Sinodo dei Vescovi:

È vero che altre questioni, gravi, vengono poste quando la mancanza di sacerdoti è avvertita in modo tragico [...]. Alcuni si sono domandati se non sia il caso, in tali circostanze, di pensare all'ordinazione di viri probati. Questa soluzione non è da prendersi in considerazione e al problema posto occorre rispondere con altri mezzi. Come è noto, la possibilità di fare appello a dei viri probati è troppo spesso evocata nel quadro di una propaganda sistematica ostile al celibato sacerdotale. Tale propaganda trova il sostegno e la complicità di alcuni mass media. Occorre quindi cercare, senza indugio, altre soluzioni a questo angoscioso problema pastorale ${ }^{102}$.

L'evangelizzazione ha bisogno di operatori preparati per la missione. Nell'Instrumenum laboris al Sinodo dei Vescovi del 2012, su "La nuova evangelizzazione per la trasmissione della fede cristiana", si trova il punto intitolato "Centralità delle vocazioni" (nn. 159-161) in cui si legge:

In questa prospettiva si aspetta che il prossimo appuntamento sinodale metta a tema in modo esplicito la centralità della questione vocazionale per la Chiesa di oggi [...] dovrà prestare un'attenzione particolare al ministero presbiteriale e alla vita consacra-

100 Cfr. M.A. Kopiec, L'evangelizzazione nel recente magistero dei papi. Tra le sfide, il mandato e la carità, Terni 2016, 275.

101 Cfr. Ma il sacerdote non è già sposato con la Chiesa? Chi è che vuole la botte piena e la moglie ubriaca?, https://cronicasdepapafrancisco.com/2019/10/16/ma-il-sacerdote-non-e-gia-sposato-con-la-chiesa-chi-e-che-vuole-la-botte-piena-e-la-moglie-ubriaca/.

102 Cfr. Giovanni Paolo II, Discorso in occasione dell'VIII Assemblea Generale Ordinaria del Sinodo dei Vescovi, 6, 27 ottobre 1990, https://w2.vatican.va/content/john-paul-ii/it/speeches/1990/ october/documents/hf_jp-ii_spe_19901027_sinodo. 
ta, auspicando che il Sinodo porti alla Chiesa il frutto di nuove vocazioni sacerdotali, rilanciando l'impegno di una chiara e decisa pastorale vocazionale ${ }^{103}$.

La domanda che qui si impone è che cosa ha fatto la Chiesa nell'arco degli ultimi anni per suscitare e far crescere le nuove e sante vocazioni, tranne un continuo discreditare il sacerdozio ripetendo il mantra del "clericalismo"? Come ha operato la Chiesa per far avvicinare i giovani a Cristo per - usando una parola tanto cara a papa Francesco - "attrazione" e "bellezza" di questa forma vita dedicata completamente a Dio, alla Chiesa e alla salvezza degli uomini?

Pare che l'attuale tattica di affrontare il problema delle vocazioni sia quella di mondanizzare e ridurre il sacramento dell'ordine attraverso un primo passo, quello di una discreta liquidazione del celibato. Secondo il cardinale Müller è evidentemente sbagliata l'idea di introdurre i "viri probati". Infatti ci sono già dei diaconi sposati. Anche se loro fossero introdotti spiega l'ex Prefetto della Congregazione per la Dottrina della Fede - dovranno rispettare la consuetudine della Chiesa anti$\mathrm{ca}$, cioè vivere in castità. Non è una qualsiasi legge che può essere cambiata a piacimento. Ma ha profonde radici nel sacramento dell'ordine. Il prete è rappresentante di Cristo sposo e ha una spiritualità vissuta che non può essere cambiata. Gli apostoli hanno lasciato tutto per andare dietro a Gesù. Cristo è il modello per i ministri, i preti. E questa cosa non può essere cambiata da spinte secolari. E nemmeno si può contraddire il Concilio Vaticano II che in "Presbyterorum ordinis", al numero 16, parla del celibato e del legame di convenienza fra chi rappresenta Cristo celibe sposo e la Chiesa ${ }^{104}$. Inoltre l'antica pratica della Chiesa finalizzata a provvedere le nuove e sante vocazioni è testimoniata dall'antico Rito romano che prevedeva le cosiddette Quattro Tempora. Questo Rito consisteva, all'inizio delle quattro stagioni, in tre giorni di digiuno, astinenza e preghiera (mercoledì, venerdì e sabato), per poter ricevere il frutto della terra (l'aspetto ecologico) e la grazia dei santi e numerosi sacerdoti (l'aspetto vocazionale) ${ }^{105}$.

Secondo il cardinale Robert Sarah, spesso si sente dire, in merito al celibato sacerdotale, che si tratta semplicemente di una disciplina di carattere storico. Tale opinione sembra essere falsa. Il celibato rivela l'essenza stessa del sacerdozio cristiano. Parlarne come di una realtà secondaria significa ferire tutti i sacerdoti del

103 “Instrumenum laboris" al Sinodo dei Vescovi del 2012, su "La nuova evangelizzazione per la trasmissione della fede cristiana”, http://www.vatican.va/roman_curia/synod/documents/rc_synod_doc_20120619 instrumentum-xiii_it.html\#Centralità delle vocazioni.

104 Cfr. P. Rodari, Il cardinale Müller: "Nemmeno il Papa può abolire il celibato dei preti»", 10.10.2019, https://ilsismografo.blogspot.com/2019/10/vaticano-il-cardinale-muller-nemmeno-il.html.

105 Cfr. L. Scrosati, Creato e vocazioni? Recuperiamo le 4 tempora, 21.10.2019, https://lanuovabq. it/it/creato-e-vocazioni-recuperiamo-le-4-tempora. 
mondo. In effetti la relativizzazione della legge del celibato sacerdotale riduce il sacerdozio a una semplice funzione ${ }^{106}$.

"Chi relativizza il celibato riduce il sacerdote a funzionario e il sacerdozio a un fare, mentre è un essere"107. Gesù ci ha insegnato che il vero sacerdote offre in sacrificio se stesso. Giovanni Paolo II e Benedetto XVI l'hanno ribadito con fermezza. Sicché, sottolinea Sarah, non c'è spazio per le deroghe, nemmeno se limitate a una sola regione ${ }^{108}$. "Fin dai primi secoli della Chiesa la legge della continenza e poi il celibato sono state considerate come di origine apostolica ${ }^{109}$. Il celibato, il sacerdozio, la Croce e la verità sono realtà strettamente connesse in Gesù che è la pietra d'inciampo"110.

Nel contesto del Sinodo sull'Amazzonia e della mancanza delle nuove vocazioni presbiteriali, la proposta di alcuni vescovi e cardinali di ricorrere a uomini sposati per il sacerdozio vuol dire trattare l'Amazzonia con "disprezzo" e "umiliazione", come se Dio in questa parte del mondo fosse incapace di chiamare giovani generosi e desiderosi di donarsi totalmente, anima e corpo, nel celibato consacrato $^{111}$. Infatti per nessuna cultura, popolo e tradizione, accettare il celibato è una cosa facile, nemmeno per i cristiani stessi.

Al termine di questo capitolo, si giunge a toccare una questione ecclesiologica di capitale importanza che costituisce un'asse o base per tutte le varie idee portate insieme al Sinodo Amazzonico, è il concetto stesso della "sinodalità" 'della' e 'nella' Chiesa. Gli effetti della sinodalità come è stata definita ed applicata dai padri sinodali con a capo i rappresentanti della chiesa tedesca e latino-americana erano - come si è visto sopra - evidenti e devastanti. Ormai si potrebbe avere l'impressione di una 'dogmatizzazione' della sinodalità nella teologia e nella vita della Chiesa. Difatti, la sinodalità intesa nel modo in cui è stata sviluppata nelle riunioni sinodali, che lo si voglia o no, porta inevitabilmente alla frammentazione della Chiesa in campo dottrinale e morale: non esiste più la Chiesa di Cristo, una, santa, cattolica ed apostolica, bensì - secondo l'immagine poliedrica della Chiesa tanto voluta ed apprezzata da papa Bergoglio - molte chiese, o meglio,

106 Cfr. R. Sarah (con N. Diat), Si fa sera e il giorno ormai volge al declino, Siena 2019, 69; cfr. A.M. Valli, Celibato dei preti/ Il cardinale Sarah: "Non creiamo un nuovo sacerdozio a misura umana", https://www.aldomariavalli.it/2019/11/04/celibato-dei-preti-il-cardinale-sarah-non-creiamo-un-nuovo-sacerdozio-a-misura-umana/.

107 R. Sarah (con N. Diat), Si fa sera e il giorno ormai volge al declino..., 69.

108 Cfr. A.M. Valli, Celibato dei preti / Il cardinale Sarah: "Non creiamo un nuovo sacerdozio a misura umana", https://www.aldomariavalli.it/2019/11/04/celibato-dei-preti-il-cardinale-sarah-non-creiamo-un-nuovo-sacerdozio-a-misura-umana/.

109 R. Sarah (con N. Diat), Si fa sera e il giorno ormai volge al declino..., 72.

110 Ibidem, 82.

111 Cfr. ibidem, 85. 
diverse comunità autonome, che, essendo ognuna di natura determinata geo-politicamente (l'idea della chiesa poliedrica al posto della Chiesa una, santa, cattolica e apostolica), creano singolarmente il proprio Credo e il proprio Symbolum definitionum et declarationumn de rebus et morum, condizionato dal contesto storico, spazio - temporale. Ovviamente non si può resistere all'impressione di trovarci di fronte ad un processo, già in atto, di luteranizzazione della Chiesa cattolica. Invece, come scrive giustamente Stefano Fontana, non esiste una vera sinodalità nella Chiesa senza l'autentica dottrina fondata sulla divina rivelazione, depositata congiuntamente nella Sacra Tradizione e nella Sacra Scrittura ${ }^{112}$ e affidata alla Chiesa sin dai tempi degli apostoli con l'effusione dello Spirito Santo nel giorno della Pentecoste ${ }^{113}$.

\section{CONCLUSIONE}

L'obiettivo di questo studio era quello di ricordare e mostrare una continua attualità dell'enciclica Redemptoris missio di san Giovanni Paolo II, in occasione del $\mathrm{XXX}^{\circ}$ anniversario della sua promulgazione nel 1990. L'importanza di questa enciclica, nonostante i 30 anni trascorsi, proprio oggi emerge con una particolare evidenza. Il suo rilievo si rende palese esattamente in questo periodo segnato da molti cambiamenti che stanno accadendo nella Chiesa e che mettono in repentaglio i punti fermi della fede e della morale della Chiesa, anzi del Vangelo che è stato affidato da Cristo agli apostoli, cioè da Dio alla Chiesa. In questo documento papa Wojtyła cercava di rammentare i temi fondamentali riguardanti la natura della Chiesa e la necessità di evangelizzazione. La Chiesa infatti di per sé è missionaria e uno dei suoi compiti principali è l'opera missionaria. In questa maniera la Chiesa realizza ed esprime la propria identità, ovvero, essere il sacramento, cioè segno e strumento della salvezza universale, di tutta la creazione. La fede, essendo condizione della salvezza proclamata e proposta dalla Chiesa, non è esclusiva proprietà dei cristiani, ma è un dono da condividere con gli altri. Infatti ogni essere umano ha il diritto di conoscere il contenuto della Buona Novella accolta, annunciata e divulgata dalla Chiesa da due millenni. Perciò la Chiesa mai si può dispensare da questo dovere di portare il lieto annuncio a tutti i popoli, nazioni, culture, società e tradizioni. Spetterà poi ad ogni uditore della Parola di Dio, in piena libertà, di prendere in modo responsabile la decisione: accettare o rifiutare il Vangelo di Cristo.

112 Concilio Vaticano II, Costituzione dogmatica sulla divina rivelazione "Dei Verbum", 9, EV 1/872-911.

113 Cfr. S. Fontana, Senza fedeltà alla dottrina non c'è sinodalità, 30.10.2019, https://lanuovabq.it/ it/senza-fedelta-alla-dottrina-non-ce-sinodalita. 
L'attuale situazione fuori e dentro la Chiesa rende questo dovere ancora più urgente $^{114}$. Prima, le difficoltà venivano piuttosto dai fattori esterni che ostacolavano la missione evangelizzatrice della Chiesa come ad esempio la diffidenza mentale o emotiva dei destinatari, sistemi ideologici che di principio vietavano l'agire missionario della Chiesa, l'ermetismo culturale e religioso dei popoli, la derisione e disprezzo dei sapienti o degli studiosi sicuri del potere e dell'autonomia della ragione umana ecc. Oggi i problemi che impediscono di proclamare con parresia $^{115} \mathrm{il} \mathrm{messaggio} \mathrm{evangelico} \mathrm{vengono} \mathrm{purtroppo} \mathrm{dal} \mathrm{dentro}$ della Chiesa. Fino a qualche decennio fa la convinzione sulla vocazione missionaria della Chiesa non era mai messa in discussione e non è stata mai sottoposta a qualsiasi tipo di dubbio o di ambiguità. Sin dalle origini della Chiesa era una cosa scontata e inconfutabile quella di portare il Vangelo in tutto il mondo. Ora invece assistiamo ad un atteggiamento "strano e nuovo" riscontrabile dentro la Chiesa stessa. Esso riguarda il fatto che lentamente e in modo sottile si sta inserendo e diffondendo sempre più il pensiero che richiede di frenare o rallentare una piena apertura e totale uscita della Chiesa verso il mondo con la Parola della salvezza, rimpiazzandola con l'idea del rispetto verso la diversità religiosa, culturale, etica ecc. Infatti da qualche anno all'interno della Chiesa si è creata e sta penetrando le menti dei cristiani una bizzarra idea, secondo cui l'evangelizzazione non deve contrastare il principio di dialogo e di tolleranza; il principio che si distingue per una esasperata e troppo irenica convinzione - quasi fosse un dogma laico - che la verità e la salvezza c'è dovunque, dappertutto ed è raggiungibile in qualunque modo. Anzi, è subentrata una nuova concezione della salvezza che ognuno può concepire a modo suo. Il pluralismo religioso de iure da decenni è già presente in teologia e si sta man mano infiltrando anche in maniera implicita, indiretta, quasi inosservabile, nel magistero ecclesiale ${ }^{116}$. Tale teoria, di origine modernista, rimuove la fede della Chiesa dal palcoscenico pubblico e le toglie il carattere

114 Cfr. Concilio Vaticano II, Costituzione dogmatica sulla Chiesa “Lumen gentium” 1, EV 1/284456.

115 "Parresia" (viene dal greco $\pi \alpha \rho \rho \eta \sigma i ́ \alpha)$ letteralmente significa "libertà di dire tutto". È frequente nel testo greco del Nuovo Testamento dove esprime il "coraggio e la sincerità della testimonianza". È stato molto usato nella tradizione cristiana, specie agli inizi, come contrario di ipocrisia. Col tempo la connotazione di "parresìa" si allarga indicando anche "imperturbabilità", "sincerità". Nelle fonti cristiane ha due significati fondamentali: "franchezza nel parlare", e "fiducia nel giudizio". Cfr. H.-C. Hahn, Franchezza, parresia, in:, Dizionario dei Concetti Biblici del Nuovo Testamento, eds. L. Coenen, E. Beyreuther, H. Bietenhard, Bologna 1976, 729-730.

116 "Il pluralismo e le diversità di religione [...] sono una sapiente volontà divina, con la quale Dio ha creato gli esseri umani": Documento sulla fratellanza umana per la pace mondiale e la convivenza, Viaggio Apostolico di Sua santità Francesco negli Emirati Arabi Uniti, 3-5 febbraio 2019, http://w2.vatican.va/content/francesco/it/travels/2019/outside/documents/papa-francesco_20190204_documento-fratellanza-umana.html. 
oggettivo, riducendola ad un atto esclusivamente privato, nonché ad una forma di sentimentalismo individuale e personale. In tale ottica, non sarebbe in qualche senso permesso se i cristiani ritenessero che non sia più necessario essere ancora missionari? ${ }^{117}$.

In questo contesto l'insegnamento della Redemptoris missio evidenzia proprio oggi, ancora più, la sua novità, sottolineando l'unicità e universalità della salvezza in Cristo e comprendendo la Chiesa come sacramento necessario della salvezza universale. Ecco la verità che la Chiesa non può non proclamare e non può mai far tacere, con la falsa scusa di evitare un presunto proselitismo o di dover considerare - a nome di un umanesimo secolarizzato - quasi sullo stesso piano, equivalenti, i fenomeni delle altre tradizioni religiose e il mistero della Chiesa e della sua natura missionaria.

\section{BIBLIOGRAFIA}

Magistero:

Concilio Vaticano II, Costituzione dogmatica sulla Chiesa “Lumen gentium”, in: Enchiridion Vaticanum 1/284-456.

Concilio Vaticano II, Costituzione dogmatica sulla divina rivelazione "Dei Verbum”, in: Enchiridion Vaticanum 1/872-911.

Concilio Vaticano II, Decreto sull'apostolato dei laici “Apostolicam actuositatem”, in: Enchiridion Vaticanum 1/912-1041

Concilio Vaticano II, Decreto sull'attività missionaria della chiesa “Ad gentes”, in: Enchiridion Vaticanum, Bologna $2002^{18}$, 1/1087-1242.

Concilio Vaticano II, Dichiarazione sulle relazioni della chiesa con le religioni non-cristiane "Nostra aetate, in: Enchiridion Vaticanum 1/853-871.

Giovanni Paolo II, Lettera enciclica "Redemptoris missio", in: Enchiridion Vaticanum 12 (1990), 547-732.

Giovanni Paolo II, Esortazione apostolica post-sinodale “Ecclesia in Europa”, 1-22, 28 giugno 2003, http://w2.vatican.va/content/john-paul-ii/it/apost_exhortations/documents/hf_jp-ii_exh_20030628_ecclesia-in-europa.html.

Giovanni Paolo II, Lettera apostolica “Tertio Millennio Adveniente”, in: Enchiridion Vaticanum 14 (1994), 1714-1820.

Giovanni Paolo II, Lettera apostolica, "Novo millennio ineunte”, 6 gennaio 2001, http://w2.vatican.va/content/john-paul-ii/it/apost_letters/2001/documents/hf_jp-ii_ apl_20010106_novo-millennio-ineunte.html.

Giovanni Paolo II, Discorso in occasione dell'VIII Assemblea Generale Ordinaria del

117 Cfr. D. Airoma, Essere (ancora) missionari dopo Abu Dhabi?, "Cristianità” 396 (2019) marzoaprile, 31-32. 
Sinodo dei Vescovi, 6, 27 ottobre 1990, https://w2.vatican.va/content/john-paul-ii/it/ speeches/1990/october/documents/hf_jp-ii_spe_19901027_sinodo.

Benedetto XVI, L'esortazione apostolica “Verbum Domini” (2010), in: Enchiridion Vaticanum 26/2218-2433.

Francesco, L'esortazione apostolica “Evangelii gaudium”, AAS 12 (2013), 1019-1137.

\section{Letteratura:}

Airoma D., Essere (ancora) missionari dopo Abu Dhabi?, in: "Cristianità" 396 (2019) marzo-aprile, 31-32.

Ambrogetti A., Il mistero dei 12. I vescovi del mondo a tavola con Giovanni Paolo II, Tau Editrice 2014.

Assemblea speciale del Sinodo dei vescovi per la regione panamazzonica, Instrumentum laboris, Amazzonia: nuovo cammini per la Chiesa per la una ecologia integrale, Vaticano, 17 giugno 2019, http:/www.sinodoamazonico.va/content/sinodoamazonico/it/ documenti/l-instrumentum-laboris-per-il-sinodo-sull-amazzonia1.html.

Cascioli R., Da Pompili a Tornielli, due giorni di parole in libertà, 23.10.2019, https:// lanuovabq.it/it/da-pompili-a-tornielli-due-giorni-di-parole-in-liberta.

Colombo D., Mandati ad gentes ieri, oggi e sempre, in: AA.Vv., La missione del redentore. Commenti all'enciclica "Redemptoris missio", 75-87.

Dizionario dei Concetti Biblici del Nuovo Testamento, eds. L. Coenen, H. Beyreuther-Bietenhard, Bologna 1976.

Documento sulla fratellanza umana per la pace mondiale e la convivenza, Viaggio Apostolico di Sua santità Francesco negli Emirati Arabi Uniti, 3-5 febbraio 2019, http:// w2.vatican.va/content/francesco/it/travels/2019/outside/documents/papa-francesco_20190204_documento-fratellanza-umana.html.

Fisichella R., I segni del giubileo. Il pellegrinaggio, la città di Pietro e Paolo, la Porta Santa, la professione di fede, la carità, l'indulgenza, Cinisello Balsamo 2015.

Fitzgerald M., Religioni in dialogo nel segno di Assisi, in: AA.Vv., La missione del redentore. Commenti all'enciclica "Redemptoris missio”, Bologna 1991, 102-107.

Fitzgerald M., Dialogo interreligioso. Il punto di vista cattolico, Cinisello Balsamo 2007.

Fontana S., Senza fedeltà alla dottrina non c'è sinodalità, 30.10.2019, https://lanuovabq. it/it/senza-fedelta-alla-dottrina-non-ce-sinodalita.

Gheddo P., L'Ad gentes in Giovanni XXIII e Giovanni Paolo II (25.05.2014), http://www. gheddopiero.it/index.php/lad-gentes-in-giovanni-xxiii-e-giovanni-paolo-ii-radio-maria-2014/.

Gheddo P., Missione senza se e senza ma. L'annuncio alle genti dal Concilio a Papa Francesco, Bologna 2013.

Gutierréz G., Müller G.L., Dalla parte dei poveri. Teologia della liberazione, teologia della Chiesa, Padova 2013, 15, 42. 
Hahn H.-C., Franchezza, parresia, in: Dizionario dei Concetti Biblici del Nuovo Testamento, eds. L. Coenen, E. Beyreuther, H. Bietenhard, Bologna 1976, 729-730.

http://www.fjp2.com/fr/jean-paul-ii/bibliotheque-en-ligne/discours/10585-to-the-participants-at-the-international-missiology-congress-held-by-the-urbanian-pontifical-university-october-7-1988.

Il sinodo per l'Amazzonia, forse una nuova religione tribale?, https://cronicasdepapafrancisco.com/2019/10/08/il-sinodo-per-lamazzonia-forse-una-nuova-religione-tribale/.

Invernizzi M., L'Europa nel magistero della Chiesa", "Christianità" 396 (2019) marzoaprile, 5-17.

Karotemprel S., Motivazioni e validità permanente della missione cristiana, in: AA.Vv., Riflessioni sulla “Redemptoris missio”, Roma 1991, 27-51.

Kaufman G., Religious Diversity, Historical Consciousness and Christian Theology, in: The Myth of Christian Uniqueness, eds. P. Knitter, J. Hick, New York 1988, 3-15.

Knitter P., Hans Küng's Theological Rubicon, in: L. Swidler, Towards a Universal Theology of Religion, New York 1978, 225-229.

Knitter P., No Other Name?, New York 1985.

Kopiec M.A., L'evangelizzazione nel recente magistero dei papi. Tra le sfide, il mandato e la carità, Terni 2016, 275.

Lemonnier M., Svolte storiche della missione, in: AA.Vv., La missione del redentore. Commenti all'enciclica "Redemptoris missio", Bologna 1991, 15-28.

Ma il sacerdote non è già sposato con la Chiesa? Chi è che vuole la botte piena e la moglie ubriaca?, https://cronicasdepapafrancisco.com/2019/10/16/ma-il-sacerdotenon-e-gia-sposato-con-la-chiesa-chi-e-che-vuole-la-botte-piena-e-la-moglie-ubria$\mathrm{ca} /$.

Martini C.M., L'Europa interpellata dalla Redemptoris missio, in: AA.Vv., Riflessioni sulla «Redemptoris missio», Roma 1991, 237-247.

Mattei R. de, Alla fine il Sinodo sull'Amazzonia entrerà nella storia come il Sinodo di Pachamama, 25.10.2019, https:/www.radioromalibera.org/cultura-cattolica/analisi-e-commenti/il-sinodo-di-pacha-mama/.

Mazzoleni G., L'eredità missionaria di Giovanni Paolo II, www.consolata.org/.../13273-leredita-missionaria-di-giovanni-paolo-ii.

Newbigin L., The Gospel in a Pluralist Society, Grand Rapids 1989, 25.

Ormas M., Umanesimo cristiano e modernità. Introduzione alle Encicliche sociali. Dalla "Rerum novarum" alla "Caritas in veritate", Città del Vaticano 2014.

Paolo VI, Esortazione apostolica postsinodale "Evangelii nuntiandi”, in: Enchiridion Vaticanum 5 (1975) 1588-1716.

Parzych-Blakiewicz K., Kopiec M.A., "Sumienie eklezjalne” w blasku Prawdy, Dobra i zbawienia, "Teologia w Polsce" 12 (2018) 2, 143-160.

Proselitismo, http://www.treccani.it/enciclopedia/proselitismo/. 
Ratzinger J., Fede, verità, tolleranza, Siena 2003.

Riccardi A., Giovanni Paolo II Santo. La biografia, Cinisello Balsamo 2014.

Rodari P., Il cardinale Müller: "Nemmeno il Papa può abolire il celibato dei preti” 10.10.2019, https://ilsismografo.blogspot.com/2019/10/vaticano-il-cardinale-muller-nemmeno-il.html.

Roest Crollius A.A., Missione e inculturazione, in: La missione del Redentore. Studi sull'enciclica missionaria di Giovanni Paolo II, eds. E. dal Covolo, A. Triacca, Torino 1992, 247-255.

Sarah R. (con N. Diat), Si fa sera e il giorno ormai volge al declino, Siena 2019.

Sarah R., Dio o niente. Conversazione sulla fede con Nicolas Diat, Siena 2015.

Scaramuzzi I., Strutture nuove e perfette non rispondono all'erosione della fede, http:// vaticaninsider.lastampa.it/vaticano/dettaglio-articolo/articolo/francesco-francis-francisco-44783/.

Scrosati L, Chiesa mondana e senza verità: come siamo arrivati fino a qui, 27.10.2019, https:/lanuovabq.it/it/chiesa-mondana-e-senza-verita-come-siamo-arrivati-fino-a-qui.

Scrosati L., Creato e vocazioni? Recuperiamo le 4 tempora, 21.10.2019, https://lanuovabq.it/it/creato-e-vocazioni-recuperiamo-le-4-tempora.

Scrosati L., Il Sinodo che ha dimenticato la salvezza delle anime, 28.10.2019, https:// lanuovabq.it/it/il-sinodo-che-ha-dimenticato-la-salvezza-delle-anime.

Seminario Urbano, La missione nella Bibbia, 16 aprile 1996, http://www.cistercensi.info/ monari/1996/m19960416.htm.

Sinodo Amazzonico, Il Documento finale: Chiesa alleata dell'Amazzonia, https://www. vaticannews.va/it/vaticano/news/2019-10/sintesi-documento-sinodo-chiesa-alleata-amazzonia.html.

Sinodo Amazonico. Il Documento finale: Chiesa alleata dell'Amazzonia, https://www. vaticannews.va/it/vaticano/news/2019-10/sintesi-documento-sinodo-chiesa-alleata-amazzonia.html.

Sinodo dei Vescovi, XIII Assemblea Generale Ordinaria, La nuova evangelizzazione per la trasmissione della fede cristiana, Instrumentum laboris, 2012, http://www.vatican. va/roman_curia/synod/documents/rc_synod_doc_20120619_instrumentum-xiii_it.html.

Smith W.C., Theology and the Worlds' Religious History, in: L. Swidler, Towards a Universal Theology of Religion, New York 1978, 51-72.

Strumia A., Come ha fatto la Chiesa a ridursi cosi?, 11.10.2019, https://anticattocomunismo.wordpress.com/2019/10/11/come-ha-fatto-la-chiesa-a-ridursi-cosi/.

Swidler L., Towards a Universal Theology of Religion, New York 1978.

Tomko J., La "Magna charta" per la missione del duemila, in: AA.Vv., La missione del redentore. Commenti all'enciclica “Redemptoris missio”, Bologna 1991, 7-12.

Tosatti M., Papa e vescovi servono il Magistero, non ricevono nuove rivelazioni neopagane, 28.09.2019, https://lanuovabq.it/it/papa-e-vescovi-servono-il-magistero-non-ricevono-nuove-rivelazioni-neopagane. 
Tosatti M., Papa e vescovi servono il Magistero, non ricevono nuove rivelazioni neopagane, 28.09.2019, https://lanuovabq.it/it/papa-e-vescovi-servono-il-magistero-non-ricevono-nuove-rivelazioni-neopagane.

Tremamunno M., L’Amazzonia non è il "polmone verde”. Né un paradiso, 18.10.2019, https://lanuovabq.it/it/lamazzonia-non-e-il-polmone-verde-ne-un-paradiso.

Ureta J.A., Il "cambio di paradigma" di Papa Francesco: continuità o rottura nella missione della Chiesa? Bilancio quinquennale del suo pontificato, San Paolo 2018.

Urosa Savino J., Amazzonia, quando parliamo di evangelizzazione?, 1.10.2019, https:// lanuovabq.it/it/amazzonia-quando-parliamo-di-evangelizzazione.

Valli A.M., Celibato dei preti / Il cardinale Sarah: "Non creiamo un nuovo sacerdozio a misura umana”, https://www.aldomariavalli.it/2019/11/04/celibato-dei-preti-il-cardinale-sarah-non-creiamo-un-nuovo-sacerdozio-a-misura-umana/.

Wolanin A., Linee attuali della theologia missionis, in: AA.Vv., Cristo Chiesa Missione. Commento alla "Redemptoris missio", Roma 1992, 42.

Zago M., Il contenuto della nuova evangelizzazione, in: Omnis Terra, aprile-giugno 1990, 104-109.

Zago M., L'urgenza di tutte le vocazioni missionarie, in: AA.Vv., La missione del redentore. Commenti all'enciclica "Redemptoris missio”, Bologna 1991, 88-91.

Zago M., Riscoprirci tutti missionari. La "Redemptoris missio": un orientamento per la lettura, www.indaco-torino.net/gens/34gens91.html [“Gen's - Rivista di vita ecclesiale" 34 (1991) 4].

Zambrano A., "In quei riti c'è il diavolo”. Parola di vescovo amazzonico, 23.10.2019, https://lanuovabq.it/it/in-quei-riti-ce-il-diavolo-parola-di-vescovo-amazzonico.

Zambrano A., “In quei riti c'è il diavolo”. Parola di vescovo amazzonico, 23.10.2019, https://lanuovabq.it/it/in-quei-riti-ce-il-diavolo-parola-di-vescovo-amazzonico.

Zambrano A., Hanno portato via il Signore! L'Amazzonia entra in chiesa con un culto pagano, 12.10.2019, https://lanuovabq.it/it/hanno-portato-via-il-signore-lamazzonia-entra-in-chiesa-con-un-culto-pagano.

Zambrano A., Il Sinodo per l'Amazzonia segue un'agenda politicamente corretta, 24.10.2019, https://anticattocomunismo.wordpress.com/2019/10/24/lagenda-politicamentecorretta-del-sinodo-sullamazzonia/.

Parole chiavi: Chiesa, missione, evangelizzazione, Vangelo, Cristo, salvezza, universalità 


\section{THE MISSIONARY NATURE OF THE CHURCH IN JOHN PAUL II'S ENCYCLICAL REDEMPTORIS MISSIO IN THE FACE OF THE VOICES OF THE AMAZON SYNOD}

\section{Summary}

The subject of the study is the theme of the missionary nature of the Church presented and shown in a new light in a deepened and adapted to the challenges of the end of the 20th century by Saint John Paul II in the encyclical Redemptoris missio. Today, a special opportunity to undertake this issue and to present it in the current historical and ecclesial context is the 30th anniversary of its announcement (December 7, 1990). The presentation of its contents and their analysis highlighted the uninterrupted continuity of Christ's missionary command and the unchanging relevance of the message contained in the document regarding the proclamation of the Gospel and the baptism of all people. The necessity of recalling this topic appears inevitably, taking into account the internal condition of the Church, as a result of which the mandate entrusted to all Christians loses its power and seriousness. As a result, due to the weakening of missionary awareness and responsibility manifesting itself within the Church, it seems necessary and absolutely essential to remind and refer to the encyclical of John Paul II in order to awaken among Catholics the awareness of the mission of announcing the Gospel given to them.

Keywords: Church, mission, evangelization, Gospel, Christ, salvation, universality

\section{MISYJNY CHARAKTER KOŚCIOŁA W ENCYKLICE REDEMPTORIS MISSIO JANA PAWŁA II W OBLICZU GŁOSÓW SYNODU AMAZOŃSKIEGO}

\section{Streszczenie}

Przedmiotem opracowania jest temat misyjnej natury Kościoła, przedstawiony i ukazany w nowym świetle, w pogłębiony i dostosowany do wyzwań końca XX wieku sposób przez św. Jana Pawła II w encyklice Redemptoris missio. Dziś szczególną okazją do podjęcia tej kwestii i ujęcia jej w aktualnym kontekście historyczno-eklezjalnym jest przypadająca 30. rocznica jej ogłoszenia (7 grudnia 1990). Ukazanie jej treści oraz ich analiza uwydatniły nieprzerwaną ciągłość misyjnego nakazu Chrystusa oraz niezmienną aktualność orędzia zawartego w dokumencie dotyczącym głoszenia Ewangelii i udzielania chrztu św. wszystkim ludziom. Konieczność przywołania tego tematu jest szczególnie konieczna, biorąc pod uwagę wewnętrzną kondycję Kościoła, w wyniku której powierzony wszystkim chrześcijanom mandat traci na swojej mocy i powadze. W efekcie, wobec osłabienia misyjnej świadomości i odpowiedzialności przejawiającej się wewnątrz 
Kościoła, zdaje się rzeczą niezbędną i bezwzględnie istotną przypomnienie encykliki Jana Pawła II celem przebudzenia się wśród katolików świadomości powierzonego im zadania i misji głoszenia Ewangelii.

Słowa kluczowe: Kościół, misja, ewangelizacja, Ewangelia, Chrystus, zbawienie, powszechność 